\title{
Hybrid Automatic-Repeat-reQuest Systems for Cooperative Wireless Communications
}

\author{
Hoang Anh Ngo, Student Member, IEEE and Lajos Hanzo, Fellow Member, IEEE
}

\begin{abstract}
Hybrid-Automatic-Repeat-reQuest (H-ARQ) has become an indispensable technique in reliable communication systems. Hence, in this paper we review the state-of-art and investigate the performance of H-ARQ in the context of cooperative wireless communications. As a novel advance, we also propose a relay-switching regime based H-ARQ scheme, which is capable of increasing the achievable system throughput as confirmed by both theory and practice. Furthermore, we evaluate the achievable upper-bound performance in the context of perfect Channel State Information (CSI). Then we proceed to quantify the performance erosion imposed by realistic CSIbased-coherent detection as well as low complexity non-coherent detection assisted cooperative H-ARQ. Finally, we propose a general design guide-lines for $H-A R Q$ aided cooperative systems.
\end{abstract}

Index Terms-ooperative communications, Hybrid-AutomaticRepeat-reQuest (H-ARQ), coherent and non-coherent detection.

\section{INTRODUCTION}

$\mathbf{O}$ VER the past three generations of mobile communications since the standardisation of GSM the achievable bitrate of mobile transceivers has improved from $9.6 \mathrm{kbit} / \mathrm{s}$ to rates above $10 \mathrm{MBit} / \mathrm{s}$. During this era there has also been a vast range of exciting developments in the field of applied signal processing algorithms designed for improving the achievable transceiver performance, which resulted in gradually approaching the attainable system capacity - at least under idealised simplifying conditions, as detailed in [1].

This is a tremendous achievement, because communications over wireless channels typically has to tolerate a substantially higher raw Bit-Error-Ratio (BER) than that in a wireline-based network due to the effects of fading and interference. In order to combat the deleterious effects of high BERs on the services offered to subscribers and to avoid the retransmissions of erroneous data blocks by the transport layer's error-control mechanism, the Automatic-Repeat-reQuest (ARQ) protocol is typically combined with channel codes at the physical layer. This results in a cross-layer operation-aided HybridARQ (H-ARQ) protocol. Along with the benefits of cooperative communications, which offers significant diversity and/or

Manuscript received April 11, 2012 revised October 31, 2012, March 10, 2013, and May 14, 2013. The research leading to these results has received funding from the European Union's Seventh Framework Programme ([FP7/2007-2013]) under grant agreement no [214625]. The financial support of RC-UK under the auspices of the UK-India Advanced Technology Centre as well as that of the China-UK Science Bridge in 4G Wireless Communications is also gratefully acknowledged.

The authors are with the School of Electronics and Computer Science, University of Southampton, Southampton, SO17 1BJ, UK (e-mail: \{han08r, lh\}@ecs.soton.ac.uk).

Digital Object Identifier 10.1109/SURV.2013.071913.00073 multiplexing gains [2]-[4], relay-aided H-ARQ arrangements potentially further improve the integrity of wireless systems.

Hence, relay-aided H-ARQ arrangements have attracted substantial research attention. For example, Zhao and Valenti [5] proposed a relay-aided network including a source, a destination and multiple relays, where the relays are capable of retransmitting the packets instead of the more distant source station. This system exhibited a significant improvement in terms of the associated energy-latency tradeoffs, when compared to conventional multihop protocols implemented as a cascade of point-to-point links. The outage probability expressions of a cooperative wireless network invoking both H-ARQ and coded cooperation amongst the nodes were derived in [6]. Furthermore, Hasan and Aygolu [7] presented a cooperative H-ARQ transmission scheme based on both superposition modulation and incremental relaying transmitting additional redundancy, while Stanojev et al. [8] investigated the energy efficiency of H-ARQ protocols both in a single-user link as well as with the assistance of relay stations. Cross-layer operation assisted and superposition coding aided multiplexed H-ARQ techniques were studied by Zhang and Hanzo [9]. By contrast, Harsini et al. [10] characterized the performance of cross-layer designs conceived for H-ARQ protocols employing adaptive modulation and coding as well as adaptive cooperative diversity. A range of other contributions related to relay-aided ARQ and H-ARQ are summarized in Table I. In order to provide further insights, the fundamental principles of cooperative communications and of the H-ARQ protocol are briefly summarized below.

\section{A. Cooperative Communications}

The basic idea of cooperative communications can be traced back to the relay channel concept proposed by Van der Meulen [2] back in 1971, which was further characterized by Cover and Gamal in [3]. In [4] Sendonaris et al. proposed the conventional relay model for multiple nodes, where the nodes transmit both their own signals as well as relay the others' signals. In [27] the authors developed an energy-efficient cooperative diversity protocol based on the classic relay channel by exploiting the space-diversity gain of distributed antennas in order to improve the achievable data rate as well as to reduce the sensitivity of the system to channel variations. Hunter and Nosratinia [28] introduced channel coding techniques for improving the attainable performance of cooperative communications. Furthermore, Dohler et al. [29] proposed the concept of Virtual Antenna Arrays (VAAs) by emulating the action of Space-Time-Block-Codes (STBC) for singleantenna-aided cooperating users. A range of other space-time 
TABLE I

MAJOR COOPERATIVE ARQ AND H-ARQ CONTRIBUTIONS.

2005 Zhao and Proposed a practical networks comprising multiple

Valenti [5] relays operating over orthogonal time slots based on a generalization of H-ARQ.

Nam et al. Proposed cooperative communication through

[11] ARQ, attaining both full-diversity and full-rate.

2006 Dianati et Proposed a node-cooperative ARQ scheme for

al. [12] wireless ad-hoc networks, which is suitable for mobile wireless channels with high and correlated frame-error profile.

Stanojev et Analyzed the average number of retransmissions al. [13] and throughput of collaborative hybrid-ARQ Type I and Chase combining protocols provided for an arbitrary number relays.

Savazzi Developed a novel analytic model to evaluate and the outage probability and tackle the problem Spagnolini of designing the minimum number of cooperat[14] ing nodes to meet a specific outage probability requirement at the destination node, as well as effective design rules tailored for the collaborative hybrid ARQ protocol in practical environments.

$\mathrm{Yu}$ et al. Analyzed and compared the data link layer packet

[15] error rate of different ARQ protocols, including the incremental relaying and the selection relaying.

2007 Krikidis [16] Proposed a distributed truncated ARQ protocol in user cooperative networks.

Mahinthan Studied the performance of adaptive relaying et al. [17] schemes in cooperative diversity systems equipped with ARQ.

Tomasin et Derive the expressions of the outage probability al. [6] for a wireless network that integrates H-ARQ and coded cooperation among nodes.

2008 Azarian et Analyzed the performance of the ARQ-dynamic al. [18] decode and forward cooperation protocol in the single-relay-dedicated scenario and the two-usercooperation scenario.

Le and Hos- Presented an analytical model for a general sain [19] ARQ cooperative diversity scheme in clusterbased multi-hop wireless networks.

Weng and Derived the optimal diversity-multiplexing-delay

Murch [20] tradeoff in the cooperative broadcast for arbitrary number of receive nodes and arbitrary maximum number of ARQ rounds.

2009 Mahinthan Proposed a cross-layer design of a wireless comet al. [21] munication system where a quadrature signalingbased cooperative diversity system employs truncated stop-and-wait ARQ for error control.

Alcaraz Investigated the performance of cooperative ARQ

and algorithms in cellular access networks.

Garcia [22]

Hasan and

Aygolu [7]

Presented a cooperative transmission scheme based on superposition modulation and incremental relaying, which is provided by a H-ARQ type feedback channel.

Stanojev et Investigated the energy efficiency of truncated $\mathrm{H}$ al. [8] ARQ protocols in a single-user link or with the assistance of a relay station.

2010 Choi et al. Proposed an adaptive multi-node incremental re[23] laying technique in cooperative communications with AF relays.

Zhang and Proposed cross-layer super position coding aided Hanzo [9] multiplexed H-ARQ techniques.

$\mathrm{Ni}$ et Proposed an adaptive distributed HARQ based

al. [24] cooperative relaying protocol, employing spatial multiplexing, space-time transmit diversity and unicast, to reduce the latency.

Narasimhan Analysed a two-user uplink interference channel et al. [25] with H-ARQ and base station cooperation.

2011 Harsini et Presented performance analysis and cross-layer al. [10] design approaches for H-ARQ protocols, employing adaptive modulation and coding and adaptive cooperative diversity.

Chaitanya Presented a retransmission scheme based on suand Lars- perposition modulation for the symmetric relaying son [26] scenario when the number of retransmissions for a data packet is limited.

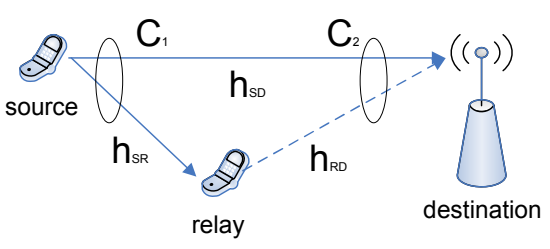

Fig. 1. Relaying topology used for a cooperative wireless network.

codes and cooperative diversity protocols were proposed by Laneman and Wornell [30] for achieving an improved spectral efficiency. Furthermore, the analysis and comparison of various cooperative strategies were detailed in [31], [32]. More recently, the soft information relaying concept was proposed in [33], [34] for improving the reliability of the relay links. On the other hand, Xiao et al. [35] introduced the concept of network coding into cooperative communications, which was further developed in [36] for mitigating the throughput loss, while attaining the maximum achievable diversity gain, which is sometimes referred to as 'full' diversity gain. In contrast to physical-layer cooperation, cooperation at the Medium Access Control (MAC) layer was described in [37].

In principle, the typical cooperative network shown in Fig. 1 contains a Source Station (SS), a single- or multiple Relay Stations (RS) and a Destination Station (DS). In the first cooperative phase, the SS broadcasts its data to both the RSs and the DS. Then, depending on the relaying type employed, the appropriate signal processing will be carried out at the RS and then the signal will be forwarded to the DS in the second cooperative phase [38]. For example, in Amplify-andForward (AF) relaying, the signal received at the relay will be amplified before being forwarded to the destination receiver. By contrast, in Decode-and-Forward (DF) relaying the relay will decode and - in the presence of errors - it would correct the errors, before re-encoding and then transmitting the stream to the DS. In order to avoid the propagation of errors to the DS, in standard DF regimes the RS only forwards the correctly decoded packets to the destination.

Finally, the multiple independently fading replicas of the signal received at the DS, which are spatially diverse are combined and recovered. Owing to the assistance of relays, typically a better link quality is expected than in a direct single-link based communication system. Additionally, the RS may reduce the signal degradation imposed by shadow-fading. Therefore, relay-aided systems are capable of increasing the attainable data rate, especially in the cell-edge region, where the MSs typically suffer both from low-power reception and from severe inter-cell interference.

\section{B. Hybrid Automatic-Repeat-reQuest Protocol}

1) ARQ Protocol: The Automatic-Repeat-reQuest (ARQ) protocol [39] constitutes a beneficial error-control method conceived for data communication over hostile channels. Its operation relies on both packet acknowledgments and time-out mechanisms. More particularly, if an acknowledgement from the receiver does not reach the transmitter before the elapse of its time-out period, the packet will be retransmitted until 
an acknowledgement is received or the predefined number of retransmissions has been exhausted.

There are three main types of the ARQ protocol, namely:

- Stop-and-wait ARQ: In this type, the transmitter sends one packet at a time and waits for the acknowledgement. If the acknowledgement is received, a new packet will be transmitted. Otherwise, a retransmission is activated.

- Go-Back-N ARQ: The transmitter continuously sends a number of packets specified by a window size of $N$. The receiver will observe the packet index. If a packet's index is not as expected, a negative acknowledgement will be returned to the transmitter, which contains this index and all the following packets will be ignored. The transmitter will retransmit the discarded packets, commencing from the most recent positively acknowledged packet. If the acknowledgement is missing, all the packets belonging to the specific window will be retransmitted.

- Selective Repeat ARQ: Unlike the Go-Back-N ARQ, this type will continue to process the packets, even if a specific packet was missing. As a result, only the specific packets, which are missing or corrupted, are retransmitted.

2) H-ARQ Protocol: In order to improve the achievable ARQ efficiency in wireless channels, the ARQ protocol is usually combined with both Forward Error Correction (FEC) and detection codes. This combination is known as the HybridARQ (H-ARQ) protocol, which was introduced in the 1960s by Wozencraft and Horstein [39], [40]. Again, it relies on both error detection and error correction combined with retransmission requests. If the channel quality is sufficiently good and all transmission errors are correctable, the receiver will request a new packet's transmission. On the other hand, if not all transmission errors may be corrected at the receiver, the received coded data block is rejected and a retransmission is requested by the receiver, similar to the basic ARQ philosophy [41]. This system is now known as the Type-I H-ARQ.

An improved version of this system, known as the TypeII H-ARQ, was invented by Lin and Yu [42], which was then modified in [43]. In the Type-II H-ARQ system, the transmitter sends additional parity bits to the receiver instead of simply retransmitting the original packet, when a retransmission request is issued. If the packet still cannot be correctly decoded, the consecutive transmissions may provide further parity information [41].

Naturally, the combination of FEC codes and the classic ARQ protocol is capable of improving the achievable throughput and of reducing the number of retransmissions, hence the delay. Type-I H-ARQ typically suffers from a throughput loss due to rejecting erroneous packets, which may be partially recoverable. By contrast, Type-II H-ARQ does not, because only additional parity bits are transmitted and combined with subsequent transmissions attempts in order to improve the probability of error-free detection. Under favourable propagation conditions, Type-II H-ARQ performs as well as standard ARQ in terms of its throughput. By contrast, in poor channel conditions, Type-II H-ARQ performs similarly to standard FEC dispensing with retransmissions.

Diverse FEC schemes may be employed in H-ARQ systems. In the early stages of development, simple channel codes,

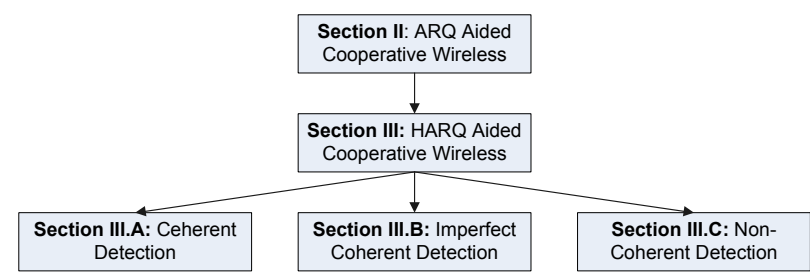

Fig. 2. Outline of our investigations

such as Golay codes or the Reed-Solomon ( ReS) codes were combined with ARQ. The development of more powerful near-capacity codes such as Turbo Codes (TC) [44] or Low Density Parity Check (LDPC) [45] rendered H-ARQ schemes more attractive in terms of improving the system's efficiency. More recently, the family of rateless codes, which are powerful erasure-filling codes, including Luby Transform (LT) codes [46] and Raptor codes [47] were also employed in H-ARQ systems for transmission over wireless networks. The H-ARQ principle is widely employed in contemporary communication systems, such as the UMTS and the 3GPP LTE standards [48] or in the IEEE 802.16 mobile WiMAX systems [49].

Due to the advantages of both the cooperative communications and the HARQ protocol, this paper will focus on analyzing the benefits of the HARQ schemes when employing in the cooperative communications. More particularly, in Section II we will study the benefits of the ARQ protocol in cooperative relaying network, while Section III will further analyze the effect of HARQ in the context of cooperative wireless network. More particularly, cooperative HARQ schemes based on both perfect- and imperfect-coherent detection as well as on noncoherent detection will be studied and compared to each other when possible. The outline of our main discussions is shown in Fig. 2.

They are followed by the summary conclusions and ideas for future research in Section IV. In each section, the analysis will be confirmed by Monte-Carlo simulations.

\section{ARQ AIDED CoOperative Wireless COMMUNICATIONS}

This section will describe the system model of ARQ aided cooperative wireless communications where both the conventional single-relay and the novel relay-switching aided schemes are employed. The theoretical capacity will be expressed to demonstrate that compared to the conventional single-relay aided scheme, the relay-switching aided scheme is capable of improving the overall system capacity. This result is further confirmed by the system simulation at the end of this section.

\section{A. System Model}

We will consider an ARQ scenario, where the SS broadcasts its data to both the RS and the DS in the first time slot and then the RS amplifies and forwards the data to the DS. It is assumed that the Source-to-Relay (SR), Source-to-Destination (SD) and Relay-to-Destination (RD) channels experience correlated 


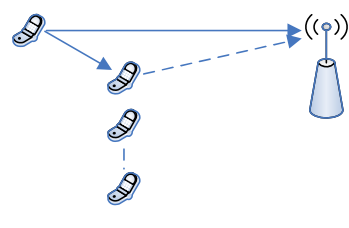

Initial Transmission

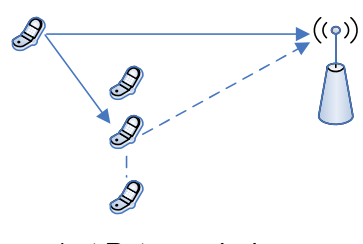

1-st Retransmission

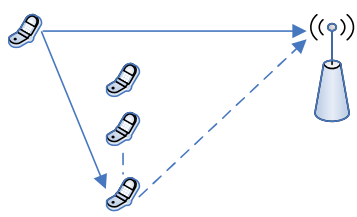

n-th Retransmission

Fig. 3. Relay-switching aided ARQ

fading, but there is no correlation between the SD and $\mathrm{RD}$ channels, owing to the substantial physical separation of the stations.

The signal received at the RS may be expressed as

$$
y_{R}[i]=G_{S R} h_{S R}[i] x[i]+n_{S R}[i],
$$

where $i$ is the symbol index; $x[i]$ is the transmit signal of the SS with power $P_{S}$ and $y_{R}[i]$ is the signal received at the RS; $n_{S R}[i]$ represents the zero-mean complex-valued Additive White Gaussian Noise (AWGN) with a variance of $\sigma_{n}^{2} ; h_{S R}[i]$ denotes the non-dispersive Channel Impulse Response (CIR) between the SS and the RS, which is modelled as a Wide-Sense Stationary (WSS) Zero-Mean Complex Gaussian (ZMCG) random process with a variance of $\sigma_{S R}^{2}$; and $G_{S R}$ is the path-loss-related power gain for the Sourceto-Relay (SR) link.

The RS amplifies $y_{R}[i]$ and forwards it to the DS, which receives Eq. 2 (next page) where, again, $y_{D}[i]$ is the signal received at the DS; $h_{S D}[i]$ and $h_{R D}[i]$ are the channel between the $S D$ and $R D$ links, which are modeled as a WSS ZMCG process with a variance of $\sigma_{S D}^{2}$ and $\sigma_{R D}^{2}$, while $n_{S D}[i]$ and $n_{R D}[i]$ are the zero-mean complex AWGN process having a variance of $\sigma_{n}^{2}$. Furthermore, $G_{S D}$ and $G_{R D}$ are the path-lossrelated power gain for the $S D$ and $R D$ links. Finally, $A_{R}$ is the fixed relay gain, which is expressed as [50]

$$
A_{R}[i]=\sqrt{\frac{P_{R}}{P\left[\left|y_{R}[i]\right|^{2}\right]}}=\sqrt{\frac{G_{S R} P_{R}}{G_{S R} P_{S} \sigma_{S R}^{2}+\sigma_{n}^{2}}},
$$

where $P_{R}$ is the transmit power of the RS.

The received packet is then decoded at the DS's receiver and then checked for the presence of residual bit errors by using error detection codes, such as the classic Cyclic Redundancy Checking (CRC) codes. If no error is detected, a positive acknowledgement is returned to the source to trigger the transmission of a new packet. Otherwise, a negative acknowledgement is fed back in order to ask for the packet's retransmission. Accordingly, the SS sends the packet again. Consequently, the combined signal received at the DS's receiver may be expressed as Eq. 4 (next page) where $L$ is the number of retransmissions, hence $L=0$ indicates that no retransmission is required.

\section{B. Single-Relay Aided $A R Q$}

The single-relay aided ARQ scheme assigns a single fixed relay to assist the SS in its transmission attempts. In mobile communications, the correlation of channel coefficients is typically reflected in the Doppler frequency, which is the change in frequency of a wave for an observer moving relative to its source [51]. When the channels experience highly correlated fading, or having high Doppler frequency, the channel coefficients change slowly according to time. This causes packet retransmission in a single path is inefficient when the deep fade occurs. As a result, there is no substantial spatial diversity gain. Nonetheless, an improved performance may be attained owing to the power-gain gleaned from the reduced-distance relaying.

\section{Relay-Switching Aided ARQ}

When the channel experiences slow fading, the single-relay aided ARQ protocol may prove to be ineffective, because the retransmitted versions may be subjected to fading, which is correlated with the versions received before. In order to further improve the attainable performance of the H-ARQ scheme considered, a novel relay-switching philosophy is proposed here. A top-level view of the scheme is illustrated in Fig. 3. According to this scheme, the SS will send the retransmitted packet through another RS every time, when a retransmission request is received. As a benefit of the independence of the channels amongst the SS, the RSs and the DS, there is no correlation amongst the signals received from the RSs at the DS's receiver. Thus, in addition to achieving a reduceddistance-based power gain, the proposed scheme also achieves a spatial diversity gain for the received signals.

\section{Capacity}

The instantaneous capacity of the relay-aided ARQ scheme may be formulated as [3]

$$
C=\min \left\{C_{1}, C_{2}\right\},
$$

where $C_{1}$ represents the sum capacity of the SD and SR links and $C_{2}$ represents the sum capacity of the RD and SD links, which are illustrated in Fig. 1.

According to the Shannon-Hartley theorem, the channel capacity $C$ is given as

$$
C=B \log _{2}\left(1+\frac{S}{N}|h|^{2}\right),
$$

where $B$ is the channel's bandwidth in Hertz and $S / N$ is the SNR at the receiver, while $h$ represents the channel's fading coefficient. For convenience, we set $B=1$ in this study and hence $B$ is dropped from the following expressions.

Substituting the Shannon-Hartley formula of Eq. (6) into our equivalent system models of Eqs. (1) and (4), the instantaneous sum capacities may be formulated as Eqs. 7 and 8 (next page).

In order to achieve the ergodic capacity, the capacities $C_{1}$ of Eq. (7) and $C_{2}$ of Eq. (7) are weighted by their specific 


$$
\begin{aligned}
y_{D}[i]= & {\left[G_{S D} h_{S D}[i] x[i]+n_{S D}[i]\right] } \\
& +\left[G_{R D} h_{R D}[i] A[i]\left(G_{S R} h_{S R}[i] x[i]+n_{S R}[i]\right)+n_{R D}[i]\right] \\
y_{D}^{L}[i]= & \sum_{l=0}^{L}\left\{\left[G_{S D} h_{S D}^{l}[i] x[i]+n_{S D}^{l}[i]\right]\right. \\
+ & {\left.\left[G_{R D} h_{R D}^{l}[i] A_{R}[i]\left(G_{S R} h_{S R}^{l}[i] x[i]+n_{S R}^{l}[i]\right)+n_{R D}^{l}[i]\right]\right\} }
\end{aligned}
$$

$$
\begin{gathered}
C_{1}=\log _{2}\left(1+\frac{P_{\text {source }} G_{S D} \sum_{l=0}^{L}\left|h_{S D}^{l}\right|^{2}}{N_{0}}+\frac{P_{\text {source }} G_{S R} \sum_{l=0}^{L}\left|h_{S R}^{l}\right|^{2}}{N_{0}}\right) \\
C_{2}=\log _{2}\left(1+\frac{P_{\text {source }} G_{S D} \sum_{l=0}^{L}\left|h_{S D}^{l}\right|^{2}}{N_{0}}+\frac{P_{\text {relay }} G_{R D} \sum_{l=0}^{L}\left|h_{R D}^{l}\right|^{2}}{N_{0}}\right)
\end{gathered}
$$

$$
\begin{array}{r}
\bar{C}_{1}=\underbrace{\int_{0}^{\infty} \int_{0}^{\infty} \ldots \int_{0}^{\infty} C_{1} \cdot F\left\{\left|h_{S D}^{0}\right|, \ldots,\left|h_{S D}^{L}\right|\right\} \cdot F\left\{\left|h_{S R}^{0}\right|, \ldots,\left|h_{S R}^{L}\right|\right\}}_{2(L+1)-\text { old }} \\
\cdot d_{S D}^{0} \ldots d_{S D}^{L} \cdot d_{S R}^{0} \ldots d_{S R}^{L}, \\
\bar{C}_{2}=\underbrace{\int_{0}^{\infty} \int_{0}^{\infty} \ldots \int_{0}^{\infty} C_{2} \cdot F\left\{\left|h_{S D}^{0}\right|, \ldots,\left|h_{S D}^{L}\right|\right\} \cdot F\left\{\left|h_{R D}^{0}\right|, \ldots,\left|h_{R D}^{L}\right|\right\}}_{2(L+1)-\text { fold }} \\
\cdot d_{R D}^{0} \ldots d_{R D}^{L} \cdot d_{S R}^{1} \ldots d_{S R}^{L}
\end{array}
$$

probability of occurrences quantified by the joint Probability Density Function (PDF) of the channel coefficients and then they are averaged - i.e. integrated over their entire dynamic range, yielding Eqns. 9 and 10, where $F\left\{\left|h_{S D}^{0}\right|, \ldots,\left|h_{S D}^{L}\right|\right\}$, $F\left\{\left|h_{S R}^{0}\right|, \ldots,\left|h_{S R}^{L}\right|\right\}$ and $F\left\{\left|h_{R D}^{0}\right|, \ldots,\left|h_{R D}^{L}\right|\right\}$ are the joint PDFs of the channel coefficients $h_{S D}^{l}, h_{S R}^{l}$ and $h_{R D}^{l}(l=$ $\{0 \ldots L\}$ is the number of retransmission), respectively.

Consequently, the ergodic capacity of the system may be formulated as

$$
\bar{C}=\min \left\{\bar{C}_{1}, \bar{C}_{2}\right\} .
$$

- Single-Relay Aided ARQ: The joint PDF of the singlerelay assisted scheme depends on the correlation of channels encountered, but more specifically on the particular correlation between the retransmitted channel envelopes at the specific instants, when the data packets were actually transmitted.

For example, in case of correlated Rayleigh fading channels and when only a single retransmission is available $(L=1)$, the joint PDF of the channel coefficients $h_{R D}^{0}$ of the first transmission and $h_{R D}^{1}$ of the retransmission for the RD link may be formulated as Eq. 12 (next page) [52], where we have $\Omega_{0}=\overline{\left|h_{R D}^{0}\right|^{2}}, \Omega_{1}=\overline{\left|h_{R D}^{1}\right|^{2}}$ and $\rho=\frac{\operatorname{cov}\left(\left|h_{R D}^{0}\right|^{2},\left|h_{R D}^{1}\right|^{2}\right)}{\sqrt{\operatorname{var}\left(\left|h_{R D}^{0}\right|^{2}\right) \operatorname{var}\left(\left|h_{R D}^{1}\right|^{2}\right)}}$ $(0<\rho<1)$ represents the channel envelope's correlation, which depends on the time-lag between two transmission attempts, i.e on the coefficients $h_{R D}^{1}$ and $h_{R D}^{2}$.

- Relay-Switching Aided ARQ: As a benefit of the relayswitching action between the retransmissions, there is no correlation between the channel coefficients of the retransmitted and original data packet. Therefore, the joint PDF of the relay switching scheme may be simplified to the product of the individual PDFs. For example, the joint PDF of transmission attempts for the RD link may be formulated as:

$$
F\left\{h_{R D}^{0}, \ldots, h_{R D}^{L}\right\}=\prod_{l=0}^{L} f\left(h_{R D}^{l}\right),
$$




$$
\begin{aligned}
F\left\{\left|h_{R D}^{0}\right|,\left|h_{R D}^{1}\right|\right\} & =I_{0}\left\{\frac{2 \sqrt{\rho}\left|h_{R D}^{0}\right|\left|h_{R D}^{1}\right|}{(1-\rho) \sqrt{\Omega_{0} \Omega_{1}}}\right\} \\
& \cdot \frac{4\left|h_{R D}^{0}\right|\left|h_{R D}^{1}\right| e^{-\left[\Omega_{0}\left|h_{R D}^{0}\right|^{2}+\Omega_{1}\left|h_{R D}^{1}\right|^{2} / \Omega_{0} \Omega_{1}(1-\rho)\right]}}{\Omega_{0} \Omega_{1}(1-\rho) \sqrt{\Omega_{0} \Omega_{1} \rho}}
\end{aligned}
$$

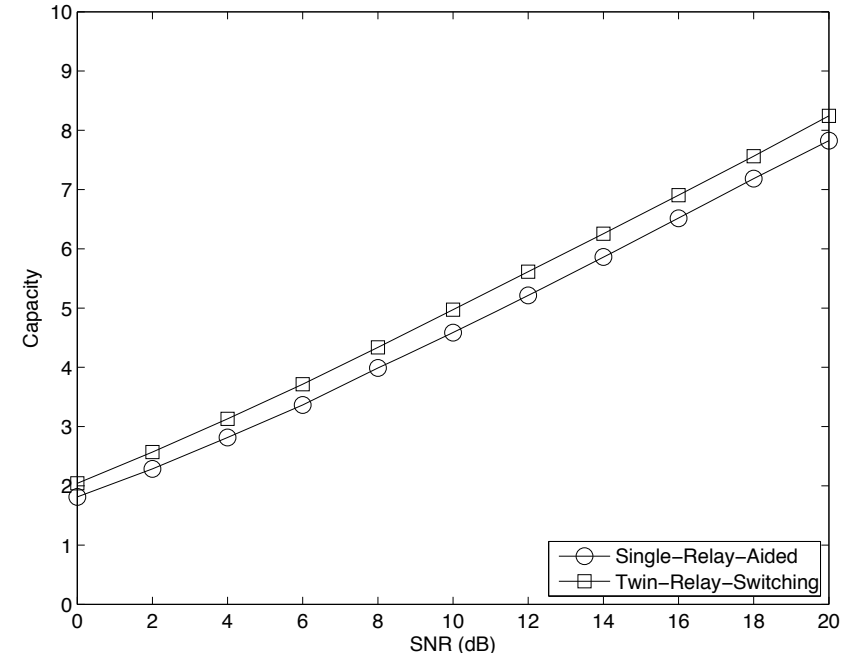

Fig. 4. Theoretical capacity of relay aided H-ARQ

where $f\left(h_{R D}^{l}\right)$ is the PDF of the Rayleigh distribution.

As a result, the joint PDF of a relay-switching scheme using two retransmissions is simplified as

$$
F\left\{h_{R D}^{0}, h_{R D}^{1}\right\}=f\left(h_{R D}^{0}\right) f\left(h_{R D}^{1}\right),
$$

Based on Eqs. (7) - (14), the theoretical capacity of both the single-relay-aided and twin-relay-aided schemes is shown in Fig. 4. This figure demonstrates that the relayswitching scheme provides a higher capacity than the singlerelay scheme.

\section{E. Performance of ARQ Aided Cooperative Networks}

In this section we characterize the achievable performance of cooperative ARQ systems of Section II-A for Quadrature Phase Shift Keying (QPSK) transmission. The relay are assumed to be in the middle of the source-destination links and equal power allocation among the stations are employed. Monte-Carlo simulation is employed and the channel follows Rayleigh distribution using Jakes' model [53] where the overall correlation is obtained via the modified Bessel function. According to Fig. 5, the single-relay-aided Decodeand-Forward (DF) cooperative ARQ achieved a power gain in excess of $5 \mathrm{~dB}$ at the maximum system throughput of 2 bits / symbol, compared to the traditional direct communication, which is shown in the continuous circle-marked curve. By contrast, the AF cooperative ARQ only achieved an improved throughput performance in the low $E_{b} / N_{0}$ region, namely below $15 \mathrm{~dB}$ for a single-relay. When the relay switching regime of Fig. 3 is employed, a further gain of approximately $2 \mathrm{~dB}$ may be gleaned in the $E_{b} / N_{0}$ region between $0 \mathrm{~dB}$ and $10 \mathrm{~dB}$, compared to the $\mathrm{AF}$ schemes. Hence, it may

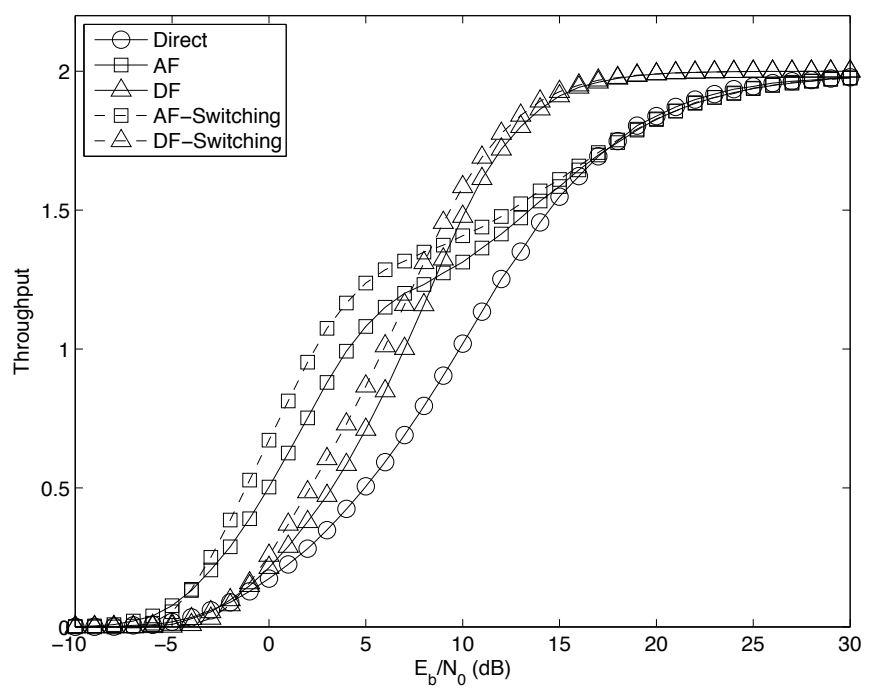

Fig. 5. The throughput of ARQ aided direct, AF and DF cooperative transmissions over correlated Rayleigh fading channels at the normalized Doppler frequency of $10^{-4}$.

be concluded that in correlated fading channels the relayswitching aided ARQ regime of Fig. 3 beneficially improves the system's performance, as confirmed by both theory and simulations in Fig. 5.

\section{HARQ AIDED COOPERATIVE WIRELESS COMMUNICATIONS}

In a further investigation, this section will consider the effects of the channel coding on the ARQ aided cooperative wireless communications, also known as the HARQ aided cooperative wireless communications. Section A will investigate the coherent-detected HARQ cooperative schemes in the context of perfect knowledge of Channel State Information (CSI). Multiple combination schemes for ARQ, namely the Chase combining [54], incremental redundancy [55] and segment selective repeat [56] will be also considered and compared. However, the perfect channel estimation, for example employing the pilot-symbol assisted scheme, is unrealistic due to the effect of noise and interference on the estimation process. Therefore, in Section B, we provide the studies of imperfect coherent detection on HARQ cooperative schemes, where different effects are considered. In contrast to the coherent detection, Section C describe the non-coherent schemes, where the redundancy components such as pilot symbols are not necessary.

\section{A. Coherent Detection for H-ARQ Aided Cooperative Wireless Communications}


1) Relay Switching Aided Turbo Coded Hybrid-ARQ in Correlated Fading Channels: Typically, the channel characteristics substantially affect the attainable system performance. For instance, correlated fading may corrupt all the consecutive retransmissions, especially when using Chase Combining (CC) [54] in H-ARQ, since the transmitted replica may also experience a deep fade. In this scenario the spatial diversity of Multiple-Input-Multiple-Output (MIMO) systems [57] may mitigate the problem. However, employing co-located MIMO elements at the Mobile Station (MS), which has compact physical dimensions, may also become ineffective in the presence of spatially correlated fading, even when employing long channel interleavers for randomizing the position of bursty error statistics. To overcome this potential drawback, we may employ distributed MIMOs relying on single-antenna based mobiles sharing their antennas by invoking cooperative relaying. We exploit the flexibility of cooperative networks [58], [59] by advocating the novel relay-switching regime detailed in Section II, where the specific relay activated is changed after each transmission attempt, in order to overcome the spatial correlation effects. Additionally, we may combine the proposed relay-switching regime with H-ARQ relying on the Segment Selective Repeat technique of (SSR) [56], where not all, but only the most error-infested segments are retransmitted, in order to further improve the overall performance of the system.

- TC-HARQ Using Chase Combining: In the H-ARQ scheme of Fig. 6, the ARQ protocol is combined with convolutional turbo coding. A CRC code is used in order to produce a positive or negative acknowledgement. More particularly, at the SS a $Q$-bit CRC code defined over the Galois Field $G F(2)$ is attached to $(K-Q)$ information bits, resulting in the bit sequence of $\mathbf{x}=\left\{x_{1}, x_{2}, \ldots, x_{K}\right\}$. This bit sequence is then passed to a $(N, K)$ turbo encoder, which turns $K$ uncoded input bits into $N$ encoded bits. The resultant bits are then punctured. Following modulation, the packet is transmitted to both the RS and the DS.

In the DS's receiver of Fig. 6, the received packet is decoded and checked for bit errors. If there is no decoding error, the SS proceeds by transmitting a new packet. Otherwise, the same packet will be retransmitted. At the receiver, the retransmitted packets are combined by Chase combining technique [54] where the packets are linearly added together before being decoded. As a result, a power gain is achieved at the receiver after each retransmission. In case of the conventional singlerelay aided scheme of Fig 1, the routes of the retransmitted packets are the same as those of the original ones. By contrast, the routes are alternated in case of the relay-switching aided scheme of Fig. 3. When the retransmitted packets arrive at the DS's decoder of Fig. 6, they are amalgamated with the previous versions using the $\mathrm{CC}$ technique of [54].

- TC-HARQ using Incremental Redundancy: In contrast to the above-mentioned TC-HARQ arrangement, in case of the TC-HARQ using Incremental Redundancy (IR), the SS will send additional parity bits, which are unknown to the DS, whenever a negative ACK is fed back. As a benefit of additional parity bits, a coding gain will be achieved at the DS's channel decoder. However, the studies of [55] demonstrated that the HARQ using IR has an inferior performance
TABLE II

THE PARAMETERS OF TC-HARQ SCHEMES

\begin{tabular}{|c|c|c|c|c|c|c|}
\hline Scheme & A-1 & A-2 & $\mathbf{A - 3}$ & B-1 & B-2 & B-3 \\
\hline$\sharp$ of infor. bits & 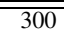 & $\overline{\overline{300}}$ & 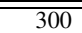 & 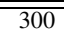 & 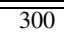 & 300 \\
\hline$\sharp$ of bits of $1^{s t}$ trans. & 600 & 600 & 600 & 600 & 600 & 600 \\
\hline$\sharp$ of bits per retrans. & 200 & 200 & 200 & 200 & 200 & 200 \\
\hline$\sharp$ of re-/IR-trans. & 3 & 3 & 3 & 3 & 3 & 3 \\
\hline Combining Type & $\mathrm{CC}$ & $\mathrm{CC}$ & $\mathrm{CC}$ & IR & $\overline{\mathrm{IR}}$ & IR \\
\hline Relaying type & none & $\overline{\mathrm{AF}}$ & $\begin{array}{r}\text { AF- } \\
\text { switch }\end{array}$ & none & $\overline{\mathrm{AF}}$ & $\begin{array}{r}\text { AF- } \\
\text { switch }\end{array}$ \\
\hline$P_{\text {source }} / P_{\text {relay }}$ & $1 / 0$ & $.5 / .5$ & $.5 / .5$ & $1 / 0$ & $.5 / .5$ & $.5 / .5$ \\
\hline$G_{s d} / G_{s r} / G_{r d}$ & $1 / 4 / 4$ & $1 / 4 / 4$ & $1 / 4 / 4$ & $1 / 4 / 4$ & $1 / 4 / 4$ & $1 / 4 / 4$ \\
\hline Scheme & $\overline{\mathrm{C}-1}$ & $\overline{\mathrm{C}-2}$ & $\overline{\mathrm{C}-3}$ & $\overline{\mathrm{D}-1}$ & $\overline{\mathrm{D}-2}$ & $\overline{\overline{D-3}}$ \\
\hline$\sharp$ of infor. bits & 500 & 500 & 500 & 500 & 500 & 500 \\
\hline$\sharp$ of bits of $1^{s t}$ trans. & 1500 & 1500 & 1500 & 1500 & 1500 & 1500 \\
\hline$\sharp$ of bits per retrans. & 500 & 500 & 500 & 500 & 500 & 500 \\
\hline$\sharp$ of re-/IR-trans. & 3 & 3 & 3 & 3 & 3 & 3 \\
\hline Combining Type & $\mathrm{CC}$ & $\mathrm{CC}$ & $\mathrm{CC}$ & SSR & SSR & SSR \\
\hline Relaying type & none & $\overline{\mathrm{AF}}$ & $\begin{array}{r}\text { AF- } \\
\text { switch }\end{array}$ & none & $\overline{\mathrm{AF}}$ & $\begin{array}{r}\text { AF- } \\
\text { switch }\end{array}$ \\
\hline$P_{\text {source }} / P_{\text {relay }}$ & $1 / 0$ & $.5 / .5$ & $.5 / .5$ & $1 / 0$ & $.5 / .5$ & $.5 / .5$ \\
\hline$G_{s d} / G_{s r} / G_{r d}$ & $1 / 4 / 4$ & $1 / 4 / 4$ & $1 / 4 / 4$ & $1 / 4 / 4$ & $1 / 4 / 4$ & $1 / 4 / 4$ \\
\hline
\end{tabular}

in comparison to that of the H-ARQ using $\mathrm{CC}$, when the systematic bits, or the original uncoded data bits,

of the first transmission attempt are corrupted by deep fades and no more systematic bits were received during the retransmission sessions. In order to avoid the above-mentioned detrimental effect, the H-ARQ scheme of Fig. 6 using IR should include part of the systematic information in the IR transmission.

Indeed, the TC-HARQ scheme of Fig. 6 using IR may achieve three different types of gains, including a) the usual relay-aided reduced-distance-based power gain, b) a spatial diversity gain achieved by the relay-switching scheme and c) channel-coding gain, which is also referred to as time-diversity gain.

- TC-HARQ Using Segment Selective Repeat: The studies provided in Chapter 5 of [60] showed that the residual decoding errors found in turbo codes usually form errorbursts, or clusters in a packet. In order to counteract this phenomenon, the authors of [56] proposed a turbo coded hybrid ARQ scheme relying on Segment Selective Repeat (SSR) for improving the attainable system performance. According to this scheme, the entire packet is divided into smaller segments, each having an identical length. Once the decoder fails, the most severely corrupted segments have to be retransmitted. In soft-decoding scenarios, our confidence in the symbolestimates is typically determined by the Log-Likelihood Ratio (LLR). The higher the LLRs, the more confident the symbol-estimates are. Therefore, the LLR values and the number of LLR polarities toggled from one iteration to another is considered to be the specific criterion for determining how severely corrupted a segment is. Hence, the segments having the lowest mean absolute LLR values and/or associated with the highest number of LLR polarity swap operations are likely to be retransmitted.

As analyzed in the previous section, the relay-switching scheme is capable of providing an increased spatial diversity gain for the ARQ scheme. Thus, it is of high potential to combine the turbo coded H-ARQ arrangement of Fig. 6 using SSR with the relay-switching scheme in order to further improve the system's performance.

In this section, we will investigate the achievable perfor- 


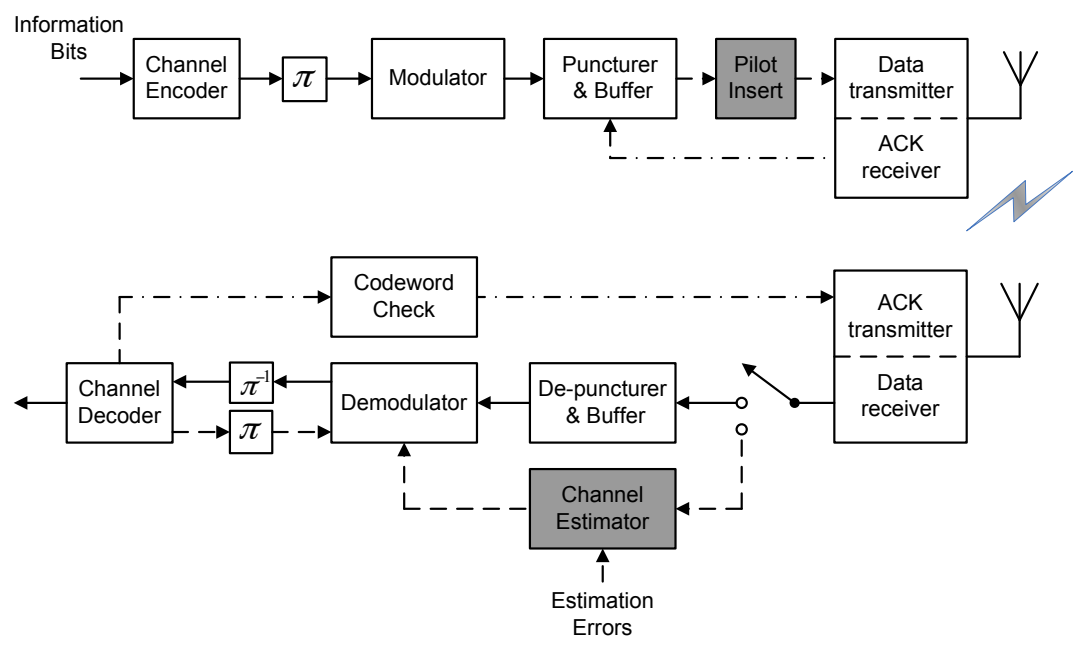

Fig. 6. The general schematic of a H-ARQ system.

mance of the relay switching aided TC-HARQ regime of Fig. 6 and compare the diverse schemes considered. The generator polynomial of the half-rate Recursive Systematic Convolutional (RSC) turbo code component is represented octally as $G(23,31)$ [61]. The CSI are assumed to be perfectly recovered.

In Scheme A-x, the first transmissions include 300 systematic bits and 300 parity bits, while the 200-bit reduced-length retransmitted packets include 100 systematic bits and 100 parity bits, as shown in Table II. Similarly, the IR transmissions of Scheme B-x contain 100 systematic bits and 100 new parity bits. The remaining parameters of each scheme are detailed in Table II. Observe in the table that the total transmit power is normalized to unity and the normalized path-loss reductions represent a relay positioned halfway between the SS and the DS. When experiencing a path-loss exponent of two, according to Fig. 7, in contrast to the direct communication schemes A1 and $\mathrm{B} 1$, a power gain of approximately $3 \mathrm{~dB}$ was achieved at the throughput value of $0.3 \mathrm{bits} / \mathrm{symbol}$, regardless, whether the single-relay-aided AF or DF schemes were employed. As a benefit of the relay-switching strategy of Fig. 3, Schemes A3 and B3 achieved a further gain of $0.5 \mathrm{~dB}$ at the same throughput value, as seen in Fig. 7.

Furthermore, we investigated the performance of the turbocoded H-ARQ of Fig. 6 using SSR in the relaying network. Again, the system parameters are provided in Table II. In the SSR schemes considered, the entire packet was divided into 15 segments, each having a length of 100 bits, where the five segments having the lowest absolute average LLR values were retransmitted. The attainable throughput performances are shown in Fig. 8, indicating that in the absence of relayswitching, relay aided H-ARQ using SSR may have a modest gain of $0.5 \mathrm{~dB}$ compared to the conventional Scheme C-2 of Table II using CC. This result is similar to that of the direct transmission Scheme C-1 characterized in Table II. By contrast, Scheme D-3, which employs SSR, may achieve an approximately $1.5 \mathrm{~dB}$ gain, when the relays are switched during the retransmission attempts. Furthermore, as seen in Fig. 8, the throughput curve of Scheme D-3 suggests that

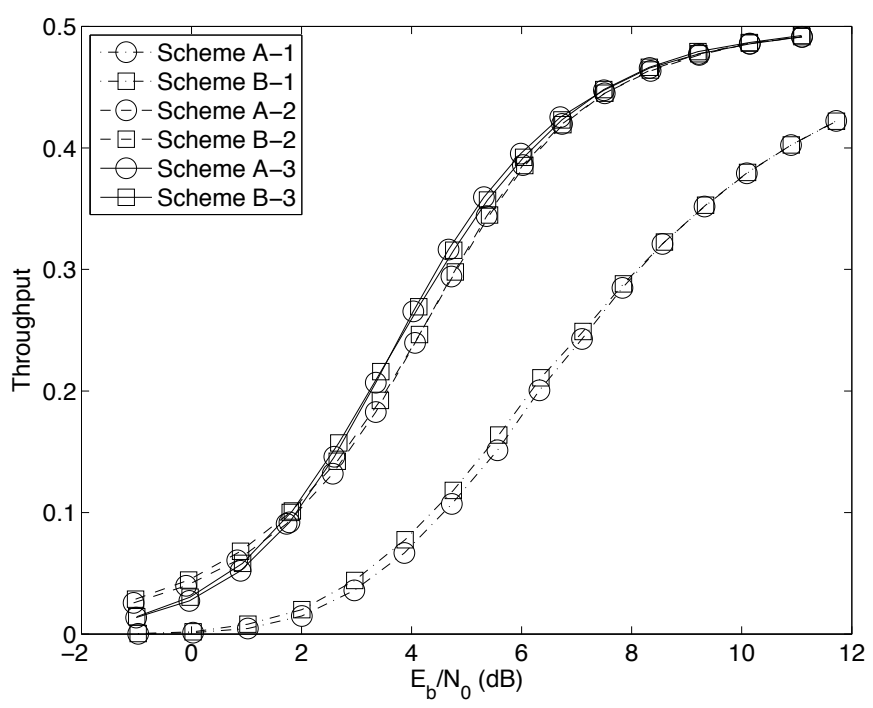

Fig. 7. The average number of retransmissions and the throughput performance of turbo coded H-ARQ of Fig. 6 employing direct, AF and DF cooperative transmissions over the correlated Rayleigh fading channels at the normalized Doppler frequency of $10^{-3}$. All other parameters are summarized in Table II.

an approximately $2 \mathrm{~dB}$ lower $E_{b} / N_{0}$ is required than that of Scheme C-3 in the $E_{b} / N_{0}$ region between $0 \mathrm{~dB}$ to $5 \mathrm{~dB}$. This implies that Scheme D-3 may increase the attainable throughput of the system.

\section{B. Imperfect Coherent Detection for H-ARQ Aided Coopera- tive Wireless Communications}

Since the channel-estimation pilots are contaminated by fading, noise and interference, the Channel State Information (CSI) estimation at the receiver is often imperfect, resulting in a degraded performance. Hence, substantial research efforts have been dedicated to characterizing the effects of imperfect detection on the system's performance, especially in the context of cooperative wireless communications. For example, Patel and Stuber [50] addressed the MIMO CE 


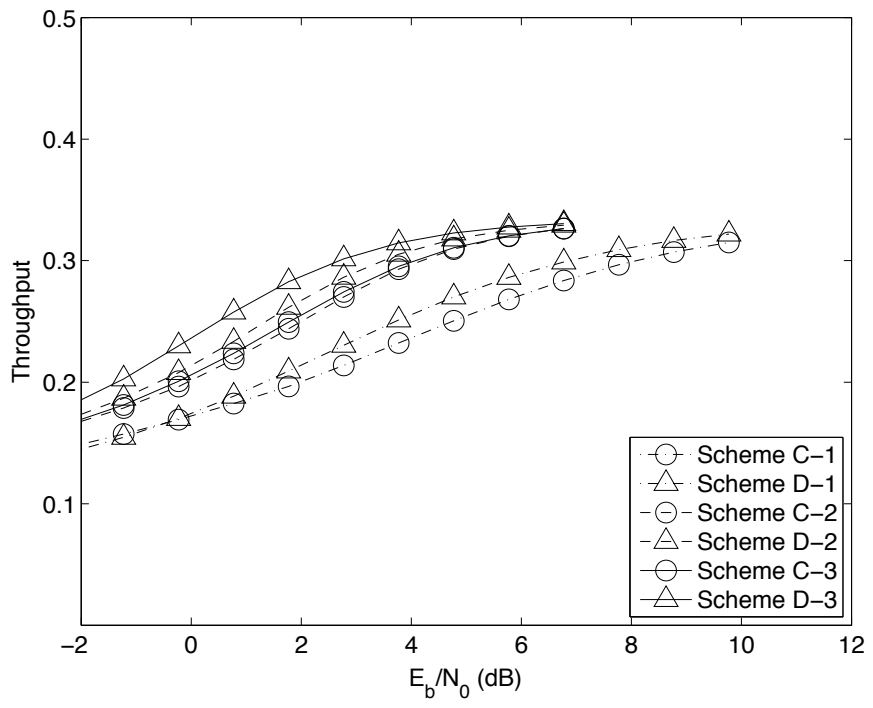

Fig. 8. The throughput performance of turbo coded H-ARQ of Fig. 6 employing direct, $\mathrm{AF}$ and $\mathrm{DF}$ cooperative transmissions over the correlated Rayleigh fading channels at the normalized Doppler frequency of $10^{-3}$. All other parameters are summarized in Table II.

design, the required pilot symbol spacing based upon realistic channel models of cooperative networks and provided an approximate BER analysis accounting for imperfect Channel Estimation (CE). Furthermore, Gedik and Uysal [62] investigated the BER performance of AF relaying in conjunction with realistic imperfect CE, while Ikki et al. [63], [64] investigated those of a cooperative network using DF relaying. Other major contributions on the imperfect-coherent detection schemes conceived for cooperative communications are summarized in Table III. These contributions, however, only considered uncoded systems, albeit all contemporary communication systems employ forward-error-correction (FEC) coding [65].

Against the above-mentioned background, in [71] we further investigated the operation of a Reed-Solomon (ReS) coded system employed in both AF and DF cooperative networks. Both the Bit Error Probability (BEP) and goodput expressions, which are defined as the ratio between the expected number of information bits that were correctly received and the number of bits transmitted in a given period of time, were derived in the context of correlated Rayleigh fading channels in order to analyze the attainable performance of the proposed system. Furthermore, the most appropriate pilot overhead and the corresponding pilot power were determined. Based on the analysis presented in [71] here we briefly characterize the achievable system performance of diverse network configurations, where the basic parameters of Table IV are employed for the schematic of Fig. 6, unless otherwise stated.

1) Effect of Doppler frequency: As mentioned in Section II, the correlation of fading channel is typically reflected in the Doppler frequency. The higher the Doppler frequency, the higher the correlation of channel coefficients. The effects of different Doppler frequencies are shown in Fig. 9. In this case, the normalized Doppler frequencies of the SR link as well as of the RD link were set to $\{0.001,0.005,0.01,0.02,0.03\}$. As detailed in [72], the pilot overhead has to be increased in order to adequately sample the increased-Doppler channels. As a
TABLE III

MAJOR CONTRIBUTIONS ON IMPERFECT-COHERENT DETECTION IN COOPERATIVE MIMO COMMUNICATIONS.

\begin{tabular}{|c|c|c|}
\hline Year & Author(s) & Contribution \\
\hline 2007 & $\begin{array}{l}\text { Patel and } \\
\text { Stuber [50] }\end{array}$ & $\begin{array}{l}\text { Addressed the MIMO CE design, the required } \\
\text { pilot symbol spacing based upon realistic channel } \\
\text { models of cooperative networks, and provided an } \\
\text { approximate BER analysis accounting for imper- } \\
\text { fect CE. }\end{array}$ \\
\hline \multirow[t]{4}{*}{2009} & $\begin{array}{l}\text { Gedik and } \\
\text { Uysal [62] }\end{array}$ & $\begin{array}{l}\text { Investigated the BER performance of AF relaying } \\
\text { with imperfect } \mathrm{CE} \text {, when a single-relay scenario } \\
\text { relying on both orthogonal and non-orthogonal AF } \\
\text { cooperative protocols was considered. }\end{array}$ \\
\hline & $\begin{array}{l}\text { Wu and Pat- } \\
\text { zold [66] }\end{array}$ & $\begin{array}{l}\text { Investigated the effects of linear MMSE CE errors } \\
\text { on the Symbol-Error-Rate (SER) of a cooperative } \\
\text { system operating in AF mode. }\end{array}$ \\
\hline & $\begin{array}{l}\text { Muhaidat } \\
\text { et al. [67] }\end{array}$ & $\begin{array}{l}\text { Investigated the effects of imperfect } \mathrm{CE} \text { on the } \\
\text { performance of distributed STBCs invoking AF } \\
\text { relaying. }\end{array}$ \\
\hline & $\begin{array}{l}\text { Han et al. } \\
{[68]}\end{array}$ & $\begin{array}{l}\text { Evaluated the BER of AF cooperative transmis- } \\
\text { sions using fixed- and variable-gain relaying in } \\
\text { the presence of imperfect CE. }\end{array}$ \\
\hline 2010 & $\begin{array}{l}\text { Ikki et al. } \\
\text { [63], [64] }\end{array}$ & $\begin{array}{l}\text { Investigated the BER performance of a coop- } \\
\text { erative network using adaptive DF relaying for } \\
\text { communication over time-selective frequency-flat } \\
\text { Rayleigh fading channels. }\end{array}$ \\
\hline \multirow[t]{2}{*}{2011} & $\begin{array}{l}\text { Zhang and } \\
\text { Gong [69] }\end{array}$ & $\begin{array}{l}\text { Investigated the impact of imperfect CSI at the } \\
\text { transmitter on the diversity gain in dynamic DF } \\
\text { relaying and proposed a power control scheme } \\
\text { based on imperfect CSI for improving the achiev- } \\
\text { able diversity gain. }\end{array}$ \\
\hline & $\begin{array}{l}\text { Ferdinand } \\
\text { and Ra- } \\
\text { jatheva [70] }\end{array}$ & $\begin{array}{l}\text { Presented the performance analysis of a two-hop } \\
\text { MIMO beamforming scheme assisted by an AF } \\
\text { fixed gain relay system using imperfect CE. }\end{array}$ \\
\hline
\end{tabular}

TABLE IV

PARAMETERS OF THE ReS CODED COOPERATIVE H-ARQ SYSTEM OF FIG. 6.

\begin{tabular}{|l|r|}
\hline Parameters & Values \\
\hline \hline $\mathrm{m} / \mathrm{k} / \mathrm{n}$ & $8 / 223 / 255$ \\
\hline Modulation type & BPSK \\
\hline Relay type & fixed \\
\hline$\sharp$ of relays & $\mathrm{L}=2$ \\
\hline Normalized distance $d_{S R_{l}} / d_{R_{l} D}$ & $0.5 / 0.5$ for AF \\
& $0.3 / 0.7$ for DF \\
\hline Data-pilot power allocation scheme & Equal \\
\hline Source's transmit power & $E_{s S}=E_{p S}=\frac{m k}{m n+N_{p}} \cdot \frac{E_{b}}{2}$ \\
\hline Relays' transmit power & $E_{s R_{l}}=E_{p R_{l}}=\frac{m k}{m n+N_{p}} \cdot \frac{E_{b}}{2 L}$ \\
\hline Channel model & correlated Rayleigh fading \\
\hline Normalized Doppler freq. of SD link & $f_{S D} T_{s}=0.002$ \\
\hline Normalized Doppler freq. of $\mathrm{SR}_{l}$ link & $f_{S R_{l}} T_{s}=0.001$ \\
\hline Normalized Doppler freq. of $\mathrm{R}_{l} \mathrm{D}$ link & $f_{R_{l} D} T_{s}=0.001$ \\
\hline Pilot oversampling factor & $L_{p}=10$ \\
\hline
\end{tabular}

consequence, the goodput of the entire system was actually reduced. Quantitatively, the goodput was reduced to about $50 \%$ for AF relaying in Fig. 9, when the normalized Doppler frequency was increased from 0.001 to 0.03 . By contrast, the goodput was only decreased to about $70 \%$ for DF relaying for the same change of the normalized Doppler frequency. The reason is that in AF relaying the number of pilot must be enough to estimate the overall Doppler frequency which are the summation of the Doppler frequencies of source-to-relay and relay-to-destination channels [50], while in DF relaying the number of pilot are required for the channel with the higher Doppler frequency [50]. 


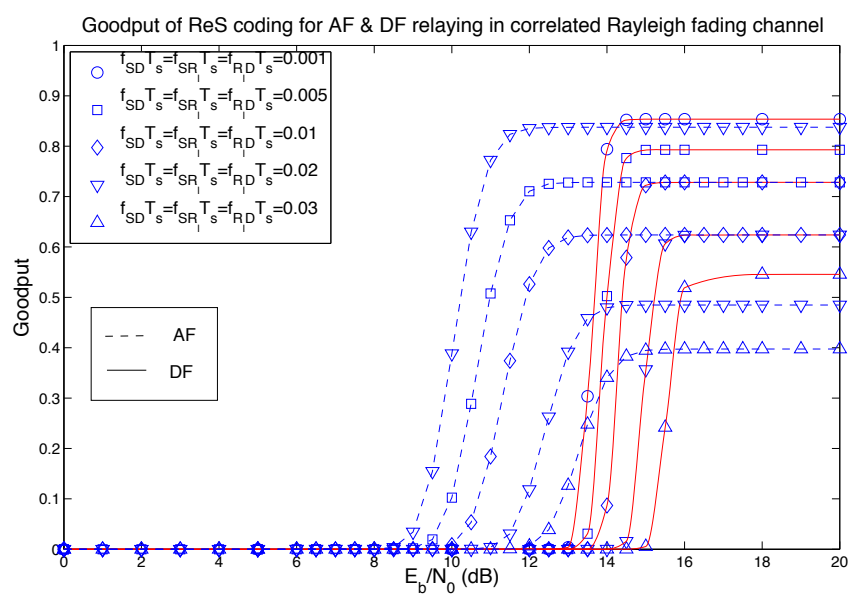

Fig. 9. Effect of different normalized Doppler frequencies on the achievable goodput in the ReS coded H-ARQ system of Fig. 6 using AF \& DF relaying, where we have $f_{S R_{l}} T_{s}=f_{R_{l}} D T_{s}=\{0.001,0.005,0.01,0.02,0.03\}$, $f_{S D} T_{s}=2 f_{S R_{l}} T_{s}$, the remaining parameters are provided in Table IV.

2) Effect of Pilot Oversampling Factor: In [50], the authors characterized the effects of both the pilot spacing and those of the specific number of pilots separately. Naturally, both of these effects may be treated jointly as those of the pilot power. Therefore, below we characterize the effects of different pilot powers. As detailed in [72], both the number of pilots and their spacing are directly related to the pilot-based channel oversampling factor, which determines how many times higher the pilot-overhead is than the minimum Nyquist-sampling rate determined by the channel's Doppler frequency. If the same power is assigned to each of the data and pilot symbols, then the effect of the pilot-based channel oversampling factor $L_{p}$ may be studied instead of the pilots' power.

It is plausible that increasing the pilot-based channel oversampling factor $L_{p}$, or - equivalently - the number of pilots, will reduce the CE Mean Square Error (MSE). However, this automatically reduces the useful data symbols' energy at a fixed total power budget. Consequently, the BEP would be increased. Hence, the optimal pilot-based channel oversampling factor $L_{o p t}$ has to be determined.

In a relaying aided mobile network, the number of available cooperating nodes, their position and channel characteristics are time-variant. Thus, the pilot-based channel oversampling factor has to be optimized for the SS. As shown in Eq. (17) of [72], the BEP is a monotonically decreasing function of the instantaneous Signal-to-Noise Ratio (SNR) $\bar{\gamma}$ at the receiver. As demonstrated in [72], the optimal oversampling factor $L_{\text {popt }}$ should be set to

$$
L_{\text {popt }}=\sqrt{\frac{\sigma_{e_{0}}^{2} \cdot m k \cdot m n \cdot \frac{E_{b}}{N_{0}}+(m n)^{2}}{m k \cdot \frac{1}{2 F_{\max } T_{p \min }} \cdot \frac{E_{b}}{N_{0}}}} .
$$

The goodput of the ReS coded AF relaying aided H-ARQ system of Fig. 6 is characterized in Fig. 10. However, in contrast to Fig. 9, Fig. 10 is now parameterized by $L_{p}$. According to the figure, the optimized value of $L_{p}$ shifts the goodput curve to the left, which is illustrated by the asteriskdashed line, but the maximum of the goodput value becomes lower than those of $L_{p}=1$ and $L_{p}=5$. This may be

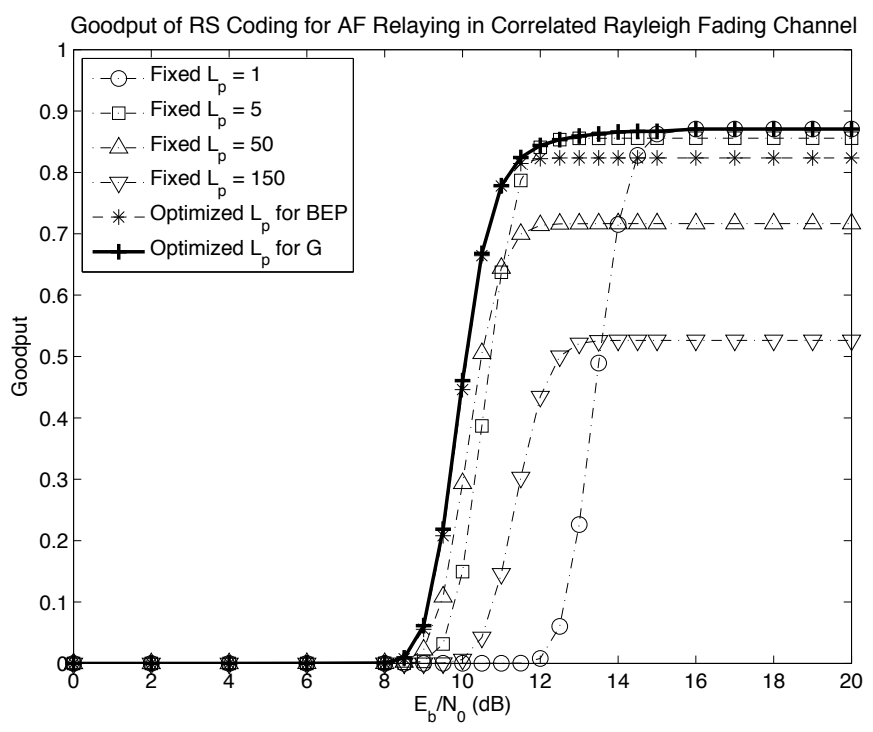

Fig. 10. Effect of the pilot oversampling factor $L_{p}$ on the achievable goodput in the ReS coded H-ARQ system of Fig. 6 using AF relaying, where we have $L_{p}=\left\{1,3,50,150, L_{\text {popt }}\right.$ for $B E P, L_{\text {popt }}$ for $\left.G\right\}$, the remaining parameters are provided in Table IV.

explained by the fact that upon minimizing the BEP by optimizing $L_{p}$, the effective rate $R_{e}$, which denotes the effective rate of each packet since the code rate of $K / N$ is further reduced by the pilots, is also reduced. Thus, the goodput of the optimized scenario characterized in Fig. 10 is also reduced. This problem may be overcome by optimizing the $L_{p}$ value in terms of goodput. The results of this optimization process are also shown in Fig. 10. Clearly, the optimized goodput curve represented by the bold continuous line in the figure indeed reaches the maximum achievable goodput value of unity.

3) Effect of the Number of Relays: In these investigations we assumed for the relay-aided network that the SS was assigned half of the total transmit power, compared to the single direct transmission, while the remaining 50\% power was equally allocated to the RSs. For a given value of $E_{b} / N_{0}$, there is an optimal number of relays. The reason for this is that when the number of RSs increases, the symbols' transmit power at the RSs has to be reduced according to our assumptions. As a result, the CE MSE increases, while the useful data symbol power decreases. As a result, the goodput may in fact be reduced, even when more relays are available. It may be observed from Fig. 11 that there is a cross-over point at $6 \mathrm{~dB}$ between the curves of the nine-relay- and fifteen-relayaided schemes. This is because in the high $E_{b} / N_{0}$ region, for example above $6 \mathrm{~dB}$, the higher-order diversity gain may compensate for the relay-power reduction, upon increasing the number of relays.

Observe in Fig. 11 that in contrast to AF relaying, in the $\mathrm{DF}$ relaying scenario the BEP always decreases, when the number of relays increases. The reason for this observation is that in the low $E_{b} / N_{0}$ region, the probability that a relay forwards a packet to the destination is low. Owing to the potential presence of errors, this procedure becomes necessary to avoid propagating more errors to the DS. When the $E_{b} / N_{0}$ is high, the relay forwards correct packets more frequently 


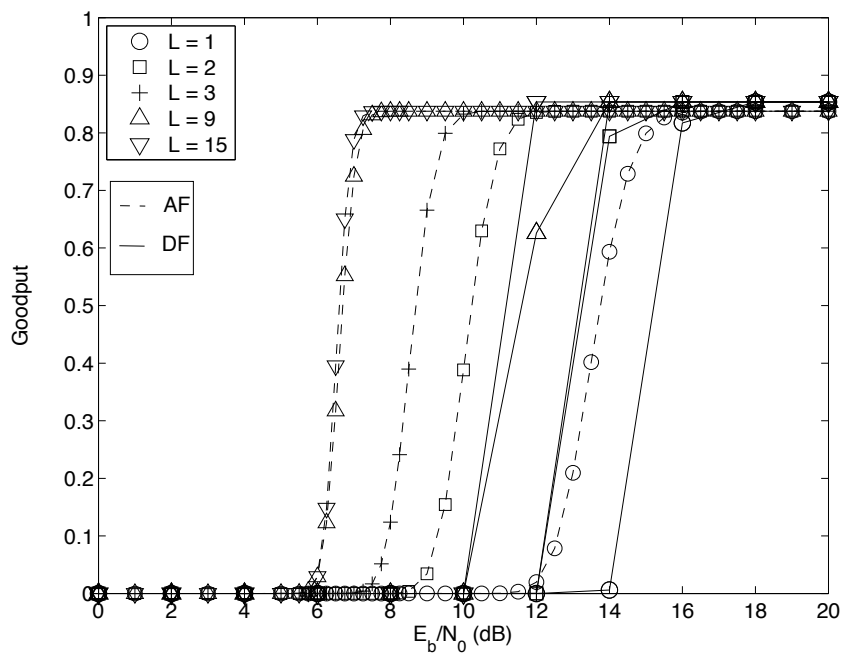

Fig. 11. Effect of the number of relays on goodput in the ReS coded H-ARQ system of Fig. 6 for AF and DF relaying: $L=\{1,2,3,9,15\}$, the remaining parameters provided as in Table IV.

and hence the BEP is reduced at the DS. Consequently, the goodput always improves commensurately with the number of assisting relays, as shown in Fig. 11.

4) Effect of Frame Length: Fig. 12 characterizes the achievable goodput for three different ReS codeword lengths, when the code rate was fixed at 0.87 . It was found from Fig. 12 that a shorter ReS codeword length of 63 symbols provided a higher goodput in the lower $E_{b} / N_{0}$ region, namely below $10 \mathrm{~dB}$, while the longer codeword of 255 symbols proved to be more efficient in the rest of the $E_{b} / N_{0}$ region. In terms of the physical interpretation of this phenomenon, in the lowSNR region even the longer and hence more potent, but more complex codes fail to correct the errors. It is widely recognized that when the longer codes are overwhelmed by an excessive number of errors, they in fact inflict a more dramatic BER degradation than their shorter counterparts. This is due to opting for the wrong legitimate long codeword, which has a higher distance from the originally transmitted codeword than a short code.

5) Effect of Code Rate: The code rate of $R=k / n$ has a substantial impact on the achievable system performance. Reducing the coding rate provides the system of Fig. 6 with a better chance of correcting the errors imposed during transmissions, but reduces the effective throughput. Therefore, selecting the most appropriate code rate is necessary. Fig. 13 characterizes the goodput of our ReS coded H-ARQ system of Fig. 6, when the code rate is varied from 0.3 to 0.98 in steps of 0.02 . It can be observed that the system performs worse, when the code rate is lower than 0.6. Depending on the channel quality, the optimal code rate may be selected from the curve, which ranges from 0.6 to 1 , as seen in Fig. 13.

The effects of system parameters are summarized in Table V. Further details of our investigations were provided in [72].

6) Comparison between $A F$ and DF Relaying: Furthermore, we compared the performance of the proposed $\mathrm{ReS} / \mathrm{H}$ ARQ system of Fig. 6 employed in the context of both AF and DF relaying networks. In relaying aided schemes, the relays'

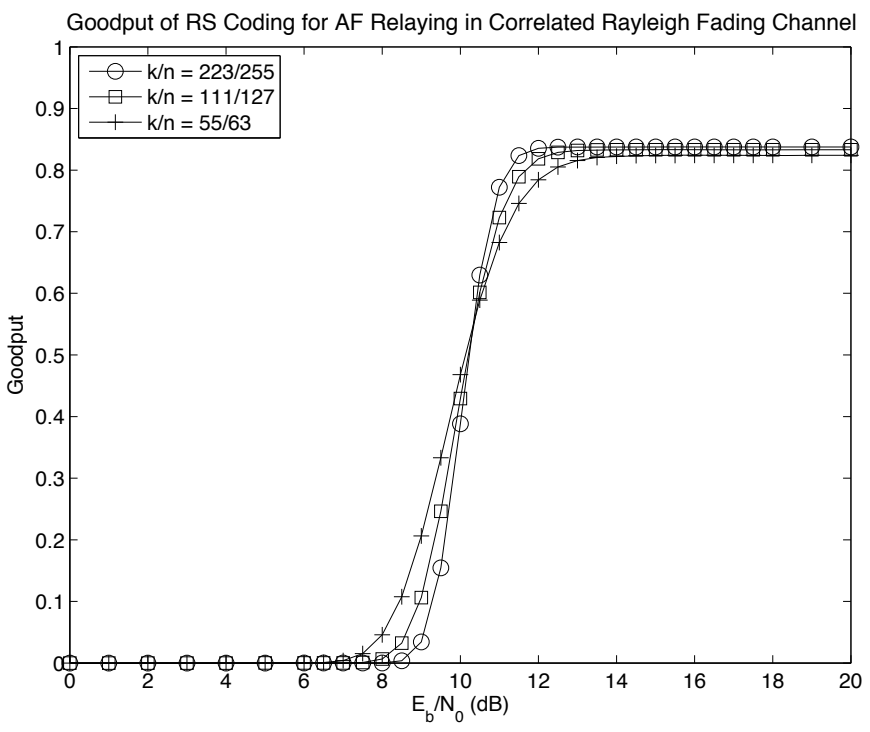

Fig. 12. Effect of codeword length on the achievable goodput in the ReS coded H-ARQ system of Fig. 6 using AF relaying where we have $k / n=\{223 / 255,111 / 127,55 / 63\}$, the remaining parameters are provided in Table IV.

Goodput of RS Coding for AF Relaying in Correlated Rayleigh Fading Channel

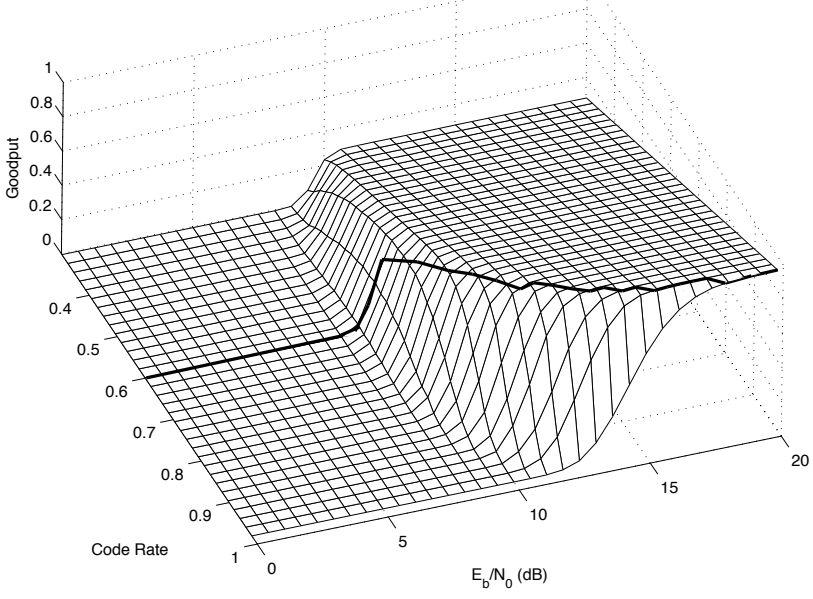

Fig. 13. Effect of code rate on the achievable goodput in the ReS coded HARQ system of Fig. 6 using AF relaying where we have $k / n=0.3 \div 0.98$ in steps of 0.02 , while the remaining parameters are provided in Table IV.

position plays an important role in determining the achievable performance at the destination. For DF relaying, the relays should be close to the source, to avoid avalanche-like error propagation, while upon roaming close to the destination, we should opt for the AF relaying scheme [73], [74]. Therefore, in these investigations we activate specific relays in those particular positions, which may be capable potentially providing the best performance for each scheme.

Our goodput results were portrayed in Fig. 14. As seen in the figure, the AF scheme may achieve a power gain of $2 \mathrm{~dB}$ at a given goodput, when compared to the DF regime. However, it becomes slightly inferior to the DF scheme in the high $E_{b} / N_{0}$ region, namely above $15 \mathrm{~dB}$. The fact is that in support of the AF scheme, the system has to use an increased proportion of pilots to deal with the detrimental effects of the combined channel, which included fading of both the SR and RD links. 
TABLE V

EFFECTS OF FACTORS ON THE PERFORMANCE OF IMPERFECT RES/H-ARQ IN COOPERATIVE NETWORKS.

\begin{tabular}{|c|c|c|}
\hline Effect & Type & Remark \\
\hline \multirow{2}{*}{$\begin{array}{l}\text { Doppler } \\
\text { frequency }\end{array}$} & $\overline{\mathrm{AF}}$ & Both the BEP and the goodput are significantly reduced when the Doppler frequency decreases. \\
\hline & $\overline{\mathrm{DF}}$ & $\begin{array}{l}\text { In the low } E_{b} / N_{0} \text { region the BER changes only insignificantly with the Doppler frequency. By contrast, in the high } E_{b} / N_{0} \\
\text { region the BER is significantly reduced, when the Doppler frequency decreases. When the Doppler frequency increases, the } \\
\text { goodput is significantly reduced due to the increase in the number of pilots required. }\end{array}$ \\
\hline \multirow{2}{*}{$\begin{array}{l}\text { Pilot } \\
\text { oversampling } \\
\text { factor }\end{array}$} & $\overline{\mathrm{AF}}$ & \multirow{2}{*}{ 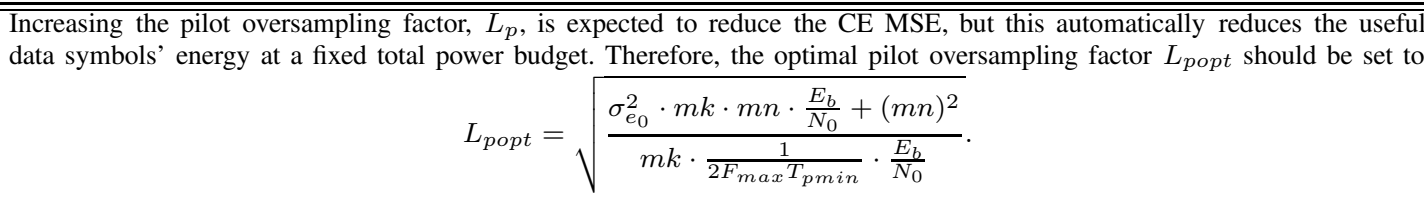 } \\
\hline & $\overline{\mathrm{DF}}$ & \\
\hline \multirow[t]{2}{*}{$\begin{array}{l}\text { Number of } \\
\text { relays }\end{array}$} & $\overline{\overline{\mathrm{AF}}}$ & $\begin{array}{l}\text { When the transmit power at the relays is identical, there is an optimal number of relays at each value of } E_{b} / N_{0} \text {, which } \\
\text { minimizes the BEP as well as maximizes the goodput. }\end{array}$ \\
\hline & $\overline{\mathrm{DF}}$ & $\begin{array}{l}\text { In contrast to AF relaying, in DF relaying the BEP and goodput always decreases, when the number of relays increases and } \\
\text { equal-power allocation is employed among the relays. }\end{array}$ \\
\hline Frame length & $\overline{\mathrm{AF}}$ & $\begin{array}{l}\text { A shorter ReS codeword provides a higher goodput in the low } E_{b} / N_{0} \text { region, while longer codewords prove to be more } \\
\text { efficient in the high } E_{b} / N_{0} \text { region. }\end{array}$ \\
\hline Code rate & 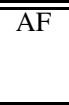 & $\begin{array}{l}\text { Reducing the coding rate provides system with a better chance of correcting errors imposed during transmissions, but reduces } \\
\text { the effective throughput. Observe that the system performs worse, when the code rate is lower than } 0.6 \text {. Depending on the } \\
\text { channel quality, the optimal code rate may be selected from the curve, which ranges from } 0.6 \text { to } 1 \text {, as seen in Fig. } 13 \text {. }\end{array}$ \\
\hline
\end{tabular}

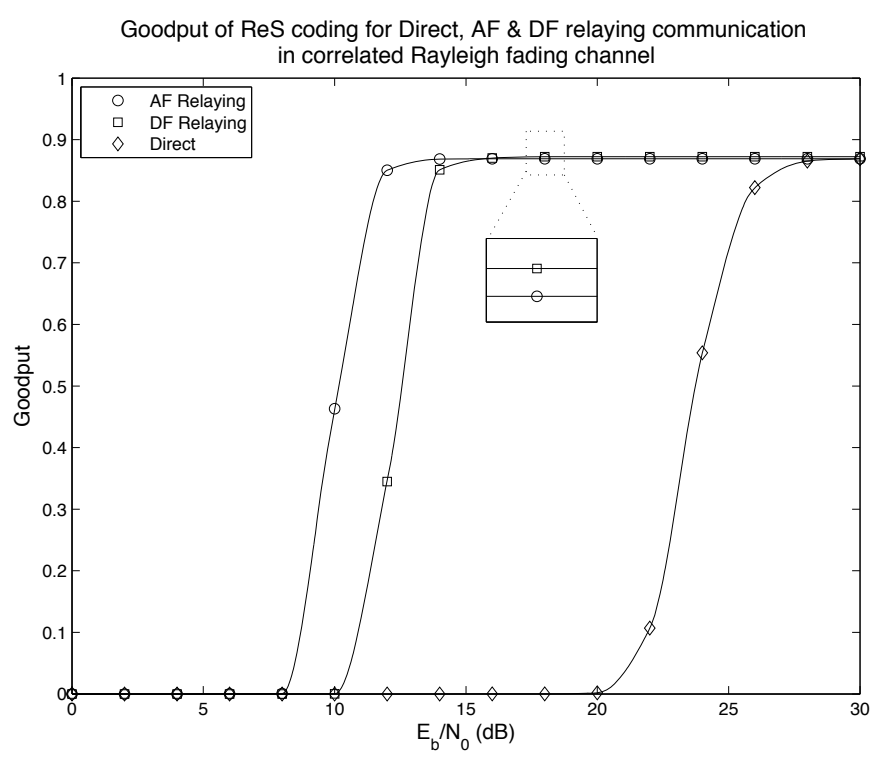

Fig. 14. Goodput versus $E_{b} / N_{0}$ of the ReS coded H-ARQ system of Fig. 6 using Direct, AF and DF relaying communications with pilot optimization, where the normalized distance of $d_{s r}=0.70$ was used for $\mathrm{AF}$, and $d_{s r}=$ 0.10 for DF relaying. The remaining parameters are provided in Table IV.

\section{Non-coherent Detection for HARQ Aided Cooperative Wireless Communications}

The HARQ schemes using coherent detection aided Pilot Symbol Assisted Modulation (PSAM) schemes may become inefficient due to the larger pilot-overhead required for channel estimation, when rapidly fluctuating fading occurs. Furthermore, channel estimation imposes a high complexity, which may become unaffordable at the relays. To overcome these impediments, non-coherent detection schemes may be considered as a design alternative [73], [74], which do not require CSI at the receiver. Non-coherent systems using differentially encoded modulation designed for cooperative networks were presented for example by Tarasak et al. [75], while Yiu et al. [76] proposed distributed Differential STBCs (DSTBC). Jing and Jafarkhani [77] further developed the differential encoding philosophy for employment in cooperative wireless communications, demonstrating that diverse space-time codes, such as Alamouti's scheme [78], the orthogonal design of [79], and the so-called $S p(2)$ design of [80], may be employed in relay-aided networks. Furthermore, Wang and Hanzo [81] proposed differentially encoded and Multiple-Symbol Differential Sphere-Detection (MSDSD) aided schemes for improving the system's performance, while reducing its decoding complexity of the classic Multiple-Symbol Differential Detector (MSDD) [82]. By contrast, Farhadi and Beaulieu [83] advocated a non-coherent low-complexity detection scheme based on M-ary Frequency Shift Keying (FSK) modulation. The salient MIMO techniques proposed for cooperative wireless communications are summarized in Table VI.

Unfortunately, all of the contributions mentioned above were investigated without the assistance of either the ARQ protocol or channel codes. Hence, in this section we will study the performance of HARQ systems employing non-coherent detection in the context of wireless cooperative networks.

\section{1) Differential Phase Shift Keying Aided H-ARQ:}

a. Differential Phase Shift Keying Relying on ReS Codes

The information-bit stream $b(i)$ is ReS encoded and then interleaved in the block $\pi$ of Fig. 6 to create the encodedbit stream $s(i)$. Then $s(i)$ is mapped to the symbols $r(i)$ before differentially encoded modulation [61], which does not require any pilot symbols for estimating the CSI. Therefore, the pilot insertion block may be omitted in Fig. 6. Instead, the assumption is that complex-valued channel envelope changes significantly slowly to exploit that any change between two consecutive received symbols was simply due to the information symbols to be transmitted. Finally, the symbols are transmitted to both the relay and to the destination.

Depending on the specific type of relaying, the signals received at the relay will be appropriately processed. If $\mathrm{AF}$ relaying is employed, the signal will be simply amplified before being forwarded to the DS. By contrast, in case of DF 
TABLE VI

MAJOR NON-COHERENT DISTRIBUTED MIMO TECHNIQUES

\begin{tabular}{|c|c|c|}
\hline Year & Author(s) & Contribution \\
\hline \multirow[t]{4}{*}{2005} & $\begin{array}{l}\text { Tarasak et } \\
\text { al. }[75]\end{array}$ & $\begin{array}{l}\text { Introduced a differentially encoded modulation } \\
\text { scheme for a two-user DF cooperative diversity } \\
\text { aided system which, does not require CSI. }\end{array}$ \\
\hline & $\begin{array}{l}\text { Himsoon et } \\
\text { al. [84] }\end{array}$ & $\begin{array}{l}\text { Proposed a differential AF transmission scheme } \\
\text { for a two-user cooperative communications sys- } \\
\text { tem. }\end{array}$ \\
\hline & $\begin{array}{l}\text { Annavajjala } \\
\text { et al. }[85]\end{array}$ & $\begin{array}{l}\text { Presented receiver structures of non-coherent } \mathrm{AF} \\
\text { communication employing on-off keying (OOK) } \\
\text { and binary FSK modulation as well as maximum- } \\
\text { likelihood (ML) detection. }\end{array}$ \\
\hline & $\begin{array}{l}\text { Yiu et al. } \\
{[76]}\end{array}$ & $\begin{array}{l}\text { Proposed differential distributed space-time block } \\
\text { coding. }\end{array}$ \\
\hline 2006 & $\begin{array}{l}\text { Wang et al. } \\
{[86]}\end{array}$ & $\begin{array}{l}\text { Designed differential distributed space-time mod- } \\
\text { ulation for cooperative networks. }\end{array}$ \\
\hline 2008 & $\begin{array}{l}\text { Jing and Ja- } \\
\text { farkhani [77] }\end{array}$ & $\begin{array}{l}\text { Designed distributed space-time coding for wire- } \\
\text { less relay networks. }\end{array}$ \\
\hline 2009 & $\begin{array}{l}\text { Oggier and } \\
\text { Lequeu } \\
{[87]}\end{array}$ & $\begin{array}{l}\text { Implemented differentially encoded distributed } \\
\text { space-time codes for an arbitrary number of relay } \\
\text { nodes. }\end{array}$ \\
\hline \multirow[t]{2}{*}{2010} & $\begin{array}{l}\text { Farhadi and } \\
\text { Beaulieu } \\
{[83]}\end{array}$ & $\begin{array}{l}\text { Proposed a low-complexity non-coherent system } \\
\text { based on M-FSK conceived for AF cooperative } \\
\text { wireless networks. }\end{array}$ \\
\hline & $\begin{array}{l}\text { Wang and } \\
\text { Hanzo [81] }\end{array}$ & $\begin{array}{l}\text { Proposed sphere-detection for the AF coopera- } \\
\text { tive UL in order to reduce complexity of classic } \\
\text { multiple-symbol differential detectors. }\end{array}$ \\
\hline
\end{tabular}

relaying the signals will be decoded and checked for errors. If errors are detected in the decoded packet, the relay will turn to the idle state. Otherwise, it will re-encode and re-modulate the packet for forwarding it to the DS.

At the destination of Fig 6, the signals received are first passed to the demodulator without requiring channel estimation. Therefore, the CE block may be omitted in Fig. 6. For classic Single Symbol Differential Detection (SSDD), the phase of the two consecutive received symbols is compared and used for determining the value of the symbols. Subsequently, the demodulated signals of both the source and of the relay are combined. In non-coherent detection, the MaximumRatio-Combining (MRC) technique cannot be invoked owing to the unavailability of the CSI. Thus, typically Equal Gain Combining (EGC) [88] and Selection Combining (SC) [88] are employed. After deinterleaving, the combined signal is decoded and checked for the presence of errors. Owing to the ReS codes' capability of fairly reliable detecting errors, the CRC-based error-detecting codes may be omitted here. If an error is detected, then the retransmissions will be activated. Otherwise, a new packet will be transmitted, as usual.

The performance of the DPSK aided ReS coded HARQ system of Fig. 6 using non-coherent detection will be investigated. The basic simulation parameters are provided in Table IV. As expected, the relaying schemes provide a better BER performance than conventional direct transmissions. Relaying also reduces the number of retransmissions, hence increasing the effective throughput, as seen in Fig. 15.

Although the Multiple-Symbol-DifferentialDetection (MSDD) technique enhances the system's resilience against increased Doppler frequencies, the decoding complexity is exponentially increased with the number of

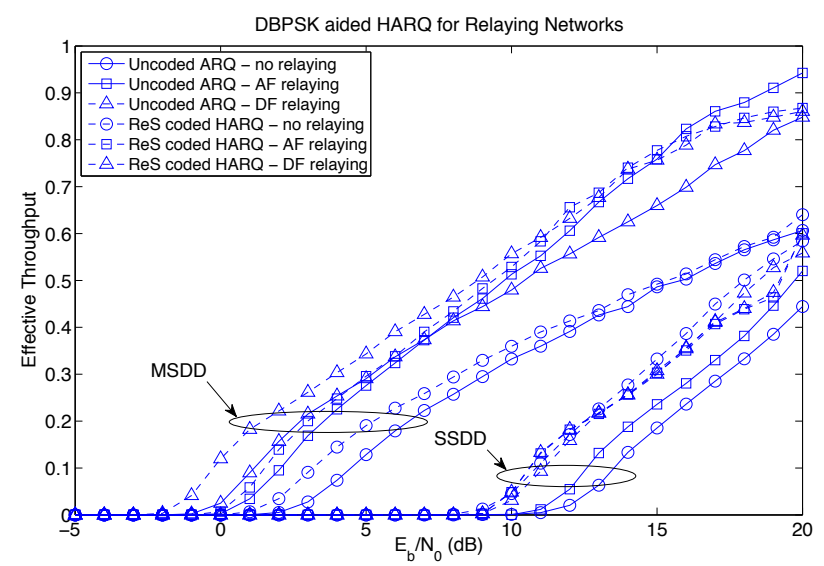

Fig. 15. Throughput of DBPSK aided HARQ for non-cooperative and cooperative networks, where the SSDD and the MSDD having a window size of 3 are employed. The other system parameters are provided in Table IV.

simultaneously detected symbols, or - synonymously - with the width of the detection window. To reduce the associated search complexity, the sphere decoding aided multiple symbol detection philosophy [89] may be combined with the MSDD scheme in the demodulator.

The conventional differential PSK is an appealingly simple detection technique, which operates without carrier acquisition and tracking in the receiver. However, it suffers from a performance penalty compared to its higher-complexity coherent counterpart. This performance penalty is further increased for high-Doppler scenarios, when the fading envelope changes substantially between the consecutive symbols. Hence, the MSDD philosophy was proposed by Divsalar and Simon [82].

For a convenient comparison between the SSDD and the MSDD schemes, all the simulation parameters in this part are the same as those of Table IV. A detection window size of $N_{w}=3$ is chosen for the MSDD.

Fig. 15 showed that the AF relaying aided schemes are capable of outperforming the DF relaying arrangements in terms of the achievable throughput in the high $E_{b} / N_{0}$ region, namely above $12 \mathrm{~dB}$ in our simulations. This observation may be explained by the fact that the MSDD receiver is capable of averaging multiple noise samples, hence the effects of noise are mitigated.

Comparing the performance of SSDD and MSDD of Fig. 15, it may be clearly recognized that the MSDD significantly improves the system's throughput compared to the SSDD scheme. Numerically, observe in Fig. 15 that the MSDD aided HARQ scheme is capable of providing a gain of up to $10 \mathrm{~dB}$ over the SSDD in terms of reducing the transmit power without degrading the system's performance .

\section{b. Differential Phase Shift Keying with Iterative Decoding}

As a further advance, we may combine the soft-output MSDD designed for DPSK with convolutional coding [90] in order to exploit the powerful principle of turbo detection as before. The transceiver schematic of the DPSK aided convolutional coded HARQ system is shown in Fig. 6, where again, a convolutional code was employed. Additionally, a Unity Rate Code (URC) having an Infinite Impulse Response (IIR) was inserted between the convolutional codec and the DPSK 


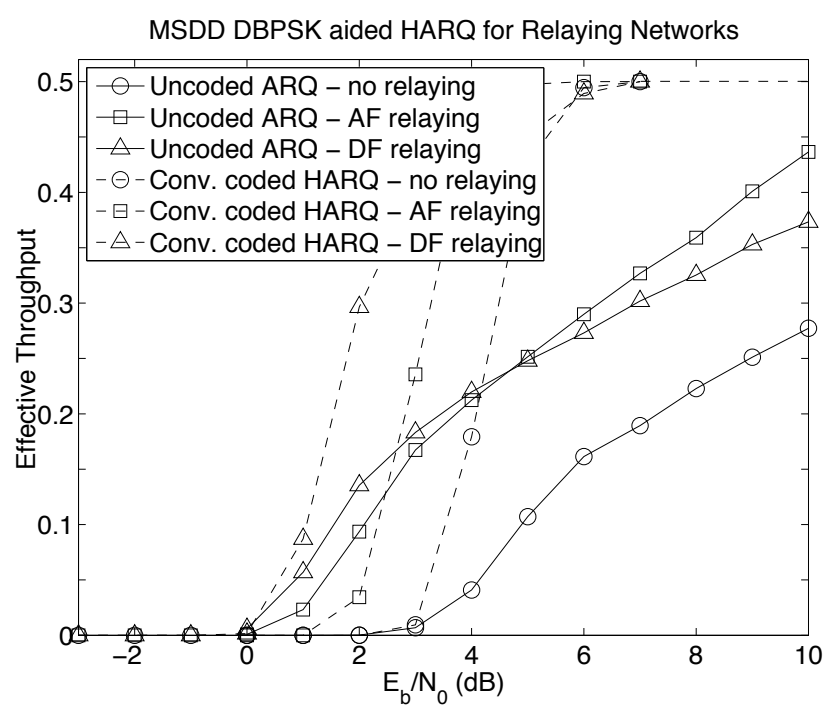

Fig. 16. DBPSK aided convolutional coded HARQ for non-cooperative and cooperative $\mathrm{AF} / \mathrm{DF}$ networks, where the system parameters are provided in Table IV.

mapper, because it was demonstrated in [90] that URCs substantially improve the convergence of iterative receivers owing to their ability to efficiently spread the extrinsic information without increasing the delay of the turbo interleaver $\pi_{1}$.

The received signals are detected with the aid of iterative information exchange between the three-detector components, namely the MSDD demapper, the URC decoder and the convolutional decoder. Following the last iteration, hard decisions are carried out and are checked for the presence of residual errors. Due to the lack of a reliable error detection capability for the family of convolution codes, an error-detecting code, such as a CRC code is concatenated with the detector. Again, the main parameters used in our simulations are shown in Table IV, where the half-rate RSC code substitutes for the ReS code.

The effective throughput seen in Fig. 16 is significantly improved, when the convolutional coding aided relaying schemes are employed. Again, observe in Fig. 16 that above the $E_{b} / N_{0}$ values of $3 \mathrm{~dB}$ and $5 \mathrm{~dB}$ for the coded and uncoded schemes, respectively, the AF relaying arrangement achieved a better throughput than the DF regime.

\section{c. Comparison of Coherent and Non-Coherent Detection}

In order to provide an insightful comparison among the perfect- and imperfect-CSI-based-coherent detection as well as the non-coherent detection schemes in the context of cooperative scenarios, we consider three cases. The first one employs coherent detection with the assistance of pilot symbols, which are assumed to be perfectly recovered at the destination's receiver. More practically, the second scenario assumed that the pilot symbols are imperfectly recovered, resulting in CSI errors at the destination's decoders. Lastly, non-coherent detection schemes using differential modulation and MSDD are also considered. As before, further details of the three schemes are provided in Table VII. The channel is assumed to have three different Doppler frequencies, namely $f_{D}=10^{-1}, 10^{-2}$ and $10^{-3}$.
TABLE VII

PARAMETERS OF CONVOLUTIONAL CODED PSK AIDED H-ARQ SCHEMES.

\begin{tabular}{|l|r|r|r|}
\hline Scheme & Perf. Coherent & Imp. Coherent & Non-Coherent \\
\hline \hline Inf./Enc. bits & $500 / 1000$ & $500 / 1000$ & $500 / 1000$ \\
\hline Channel code & RSC & RSC & RSC \\
\hline Generator poly. & $(5,7)$ & $(5,7)$ & $(5,7)$ \\
\hline Mod. type & BPSK with & BPSK with & DBPSK \\
& Pilot Symbols & Pilot Symbols & \\
\hline Demod. type & $\begin{array}{r}\text { Coherent with } \\
\text { perfect CSI }\end{array}$ & $\begin{array}{r}\text { Coherent with } \\
\text { imperfect CSI }\end{array}$ & $\begin{array}{r}\text { MSDD with } \\
W_{d}=3\end{array}$ \\
\hline$\sharp$ of pilots & $1000 \cdot 2 \cdot f_{D}$ & $1000 \cdot 2 \cdot f_{D}$ & N/A \\
\hline Detection type & Iterative & Iterative & Iterative \\
\hline Relay type & AF & AF & AF \\
\hline$\sharp$ of relays & 1 & 1 & 1 \\
\hline$d_{S R} / d_{R D}$ & $0.7 / 0.3$ & $0.7 / 0.3$ & $0.7 / 0.3$ \\
\hline Source's power & $E_{S}=\frac{E_{b}}{G_{r d}+1}$ & $E_{S}=\frac{E_{b}}{G_{r d}+1}$ & $E_{S}=\frac{E_{b}}{G_{r d}+1}$ \\
\hline Relays' power & $E_{R}=\frac{G_{r d} E_{b}}{G_{r d}+1}$ & $E_{R}=\frac{G_{r d} E_{b}}{G_{r d}+1}$ & $E_{R}=\frac{G_{r d} E_{b}}{G_{r d}+1}$ \\
\hline Fading model & correlated & correlated & correlated \\
& Rayleigh & Rayleigh & Rayleigh \\
\hline$\sharp$ of retrans. & 5 & 5 & 5 \\
\hline
\end{tabular}

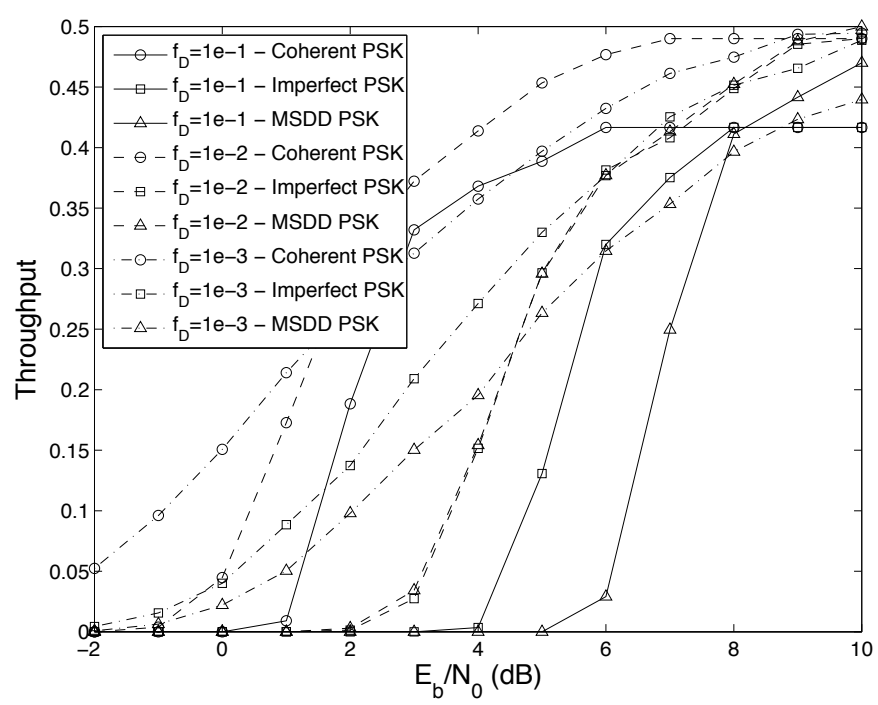

Fig. 17. DBPSK aided convolutional coded HARQ for non-cooperative and cooperative AF/DF networks, where the system parameters are provided in Table VII.

The effective throughput of the three detection schemes of Table VII is shown in Fig. 17. As seen in the figure, for the rapidly time-varying fading channels coherent detection should be employed in the low $E_{b} / N_{0}$ region, while the non-coherent MSDD should be utilized in the high $E_{b} / N_{0}$ region in order to avoid reducing the system's throughput due to the employment of a large number of pilot symbols. In moderately correlated fading channels, i.e. for $f_{D}=10^{-2}$ in the above-mentioned scenarios, the non-coherent MSDD should be chosen due to its ability to perform comparably to the coherent scheme, especially, since it is capable of providing an increased throughput for $E_{b} / N_{0}$ values above $6 \mathrm{~dB}$. By contrast, in slow-fading channels, the transmission schemes based on coherent detection have the edge.

2) Frequency Shift Keying Aided H-ARQ: When the signal travels through different paths to the receiver, frequency selective fading occurs [51], which may render non-coherent 
differential detection ineffective.

In order to mitigate the effects of frequency-selective fading channels, contemporary communication systems employ a variety of signal processing techniques, such as transmit preprocessing, channel coding and interleaving in conjunction with OFDM [90]. In [91] Garg and Adachi demonstrated that OFDM systems relying on rate-compatible punctured turbo coded HARQ are capable of signicantly reducing the number of retransmissions compared to conventional ARQ based OFDM. This is because rate-compatible codes are capable flexibly adjusting their code-rate for the sake of providing exactly the required amount of redundancy for ensuring decoding success at the destination, which hence reduces the number of retransmissions. As a further advance, Shin et al. [92] designed an OFDM aided cooperative space-time diversity assisted system, which provided as much as $6.7 \mathrm{~dB}$ energy savings over a single-antenna assisted OFDM system at a target FER of $10^{-2}$ for transmission over frequency selective fading channels. OFDM systems have recently found favour in numerous standards, such as the WiFi and WiMax systems, in the Third-Generation Partnership Project's Long-Term Evolution (3GPP-LTE), as well as in Digital Audio Broadcasting (DAB) and in Digital Video Broadcasting (DVB). However, the family of OFDM based systems imposes a potentially high detection- and channel estimation complexity. This may be acceptable in non-cooperative systems, but in cooperationaided systems it may be unrealistic in both complexity and power-dissipation terms to expect of the relays to altruistically estimate the source-relay channel for the sake of coherent detection. It would also pose a security/privacy risk to allow the relays to estimate the source-relay channel. Furthermore, OFDM systems also exhibit a high peak-to-average-power ratio, hence requiring high-linearity power-amplifiers, which tend to have a low power-efficiency.

In contrast to high-complexity coherent-detection aided non-cooperative OFDM, in this treatise we focussed our attention on HARQ-assisted cooperative communications and hence we invoked a low-complexity non-coherently detected cooperative FSK scheme, which dispenses with channel estimation. This low-complexity non-coherent FSK arrangement has a low number of parameters, which allows us to portray the HARQ characteristics and benets without having to consider a large number of OFDM modem-parameters. By contrast, adaptive OFDM systems have a large number of parameters, including the number of subcarriers, the number of subbands, the number of bits/subcarrier in each subband, the number of pilot symbols, the length of the cyclic prefix or zero-padding, the type detector, etc. Our goal was hence to avoid the complex parameterization and the associated large-scale performance study of a powerful OFDM system. Therefore again, we opted for using FSK, which may be beneficially combined with frequency hopping $(\mathrm{FH})$ for the sake of improving the achievable diversity gain as well as for supporting multiple users with the aid of uniques, user-specific FH-patterns [51]. Furthermore, FSK can be employed in the context of Space-Time-Frequency Shift Keying (STFSK) [94] for MIMO communications. As detailed in [94], the STFSK scheme improves the system's BER performance, whilst reducing its detection complexity, hence its total power dissipation. a. Reed-Solomon Coded Non-coherent Frequency Shift Keying

To elaborate further, FSK [61] constitutes a low-comlexity non-coherent detection scheme, which has benefits in the context of wireless cooperative networks. In order to mitigate the performance degradation imposed by the channel noise, interference and fading, FSK may be combined both with FEC, such as the ReS codes, as well as with the Slow Frequency Hoping (SFH) Spread Spectrum (SFH/SS) concept [95]-[98]. Owing to the FSK's capability of detecting erased and corrupted symbols, the 'error-and-erasure' decoding is preferable to the 'error-correction-only' decoding in the context of ReS coded SFH/SS systems employing $M$ ary FSK modulation, since a reduced BER may be achieved, provided that a reliable erasure insertion scheme is invoked. This is because as detailed in [51], an $\operatorname{ReS}(N, K)$ code is capable of correcting $t=\left\lfloor\frac{N-K}{2}\right\rfloor$ symbol errors, or alternatively, it can fill $2 t=(N-K)$ erased symbols provided that the receiver can confidently inform the ReS decoder, which symbols were erased by the channel. Several erasure insertion (EI) techniques assisting error-and-erasure ReS decoding were proposed in [99], [100], namely the RatioThreshold Test (RTT), the Output Threshold Test (OTT), and the joint Maximum Output and Ratio Threshold Test (MORTT).

- Ratio-Threshold Test: The RTT was proposed by Viteri [99] in order to mitigate the effects of tone and partial band jamming imposed on FSK systems. In this test the filter outputs of the M-FSK demodulator are sorted in descending order, yielding

$$
Y_{\max }=Y_{1}>Y_{2}>\ldots>Y_{M-1}>Y_{M}=Y_{\min } .
$$

Then a pre-set threshold, $\lambda_{T}$, is chosen and the ratio, $\lambda=$ $Y_{2} / Y_{1}$, of the 'second' largest decision variable is compared to the maximum to decide a decision-reliability bit $Q$, as follows

$$
Q= \begin{cases}0: & \lambda_{T} \geq \lambda(\text { high }) \\ 1: & \lambda_{T}<\lambda(\text { low })\end{cases}
$$

where $Q=0$ and $Q=1$ indicate a high- and low-reliability detection, respectively.

- Output Threshold Test: In contrast to the RTT, the OTT [101] simply makes decisions based on the maximum of the demodulator output, which is defined as

$$
Y_{1}=Y_{\max }=\max \left\{U_{1}, U_{2}, \ldots, U_{M}\right\} .
$$

Therefore, it is not the optimum decision metric. Nonetheless, the results of [101] showed that the OTT may outperform the RTT, when communicating over the dispersive channels, despite its simplicity.

- Joint Maximum Output and Ratio Threshold Test: The MO-RTT proposed by Yang and Hanzo [100] is a further development of the RTT and the OTT. In the context of the joint MO-RTT the erasure detection is based on both the observation of the maximum $Y_{1}$ and the ratio $\lambda$.

The system considered here consists of a SS, a DS and $L=1 \mathrm{DF}$ RS at the mid-way position, communicating over Rayleigh fading channels, which are additionally subjected to Partial-Band Gaussian Interference (PBGI). The cooperative communication process is divided into two time slots. In the 


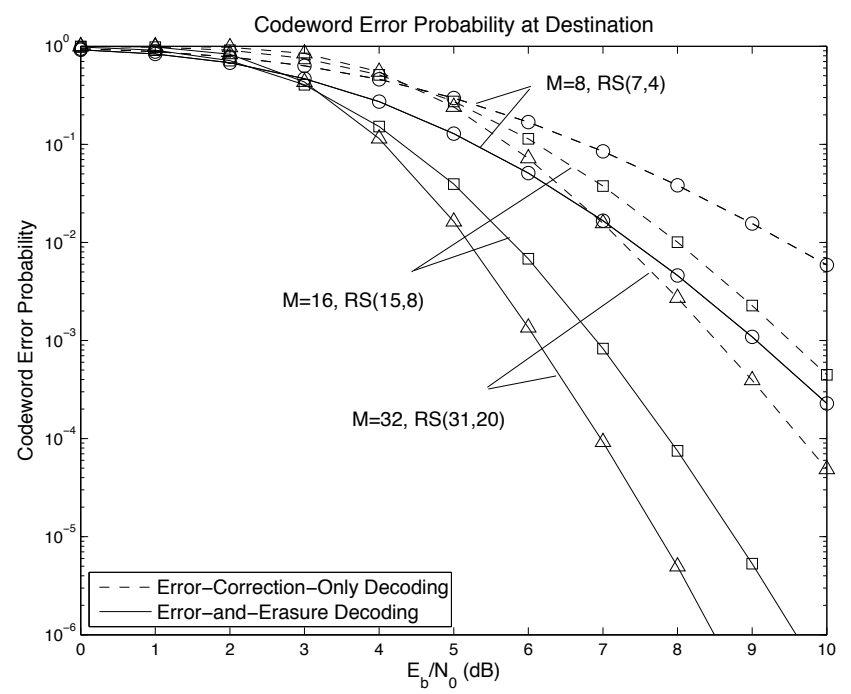

Fig. 18. Codeword decoding error probability at the destination for the various ReS FEC coded systems obeying the schematics of Figs. 1 and 6 using both "error-correction-only" and "error-and-erasure" decoding based on the MO-RTT erasure insertion scheme for transmission over frequencyselective fading channels. The results were evaluated using the parameters of $\rho=0.15, E_{b} / N_{I}=10 d B, L=1, G_{s r}=G_{r d}=4 G_{s d}$, while the channels obeyed the 4-tap COST-207 model for rural area [102].

first slot, the SS broadcasts its packets to all the RSs and the DS. In the second slot, each RS will forward the reencoded packet to the DS, provided that it correctly decoded the received information. Otherwise, the packet will not be forwarded by the RS.

Fig. 18 compared the performance of the erasure-aided and error-correction-only decoding schemes, when different FSK/ReS schemes are employed. As anticipated, the erasure insertion schemes outperform the error-correction-only arrangements. When employing the $\operatorname{Re} S(31,20)$ code combined with 32-FSK modulation and a single relay, the error-anderasure decoding scheme achieved $3 \mathrm{~dB}$ gain at the BER of $10^{-4}$ over the error-correction-only arrangement. Moreover, the ReS codes constructed over high order GFs and combined with high-order FSK modulation performed significantly better in the high- $E_{b} / N_{0}$ region, namely above $5 \mathrm{~dB}$, than those using a lower-order GF combined with FSK modulation.

Finally, we compare the performance of the proposed ReS coded scheme using error-and-erasure decoding to that of other coding schemes, such as the classic convolutional and Low Density Parity Check (LDPC) [45] codes in the context of wireless cooperative networks. The $\operatorname{RSC}(23,33)$ code using the octally represented generator polynomials of 23 and 33 [61] is considered and a regular LDPC code [45] having the same code rate and codeword length is employed. For a fair comparison, the same coding rate and the same packet size are employed for all three coding schemes. For the RSC code and the LDPC code, iterative detection is employed at the cost of a potentially increased complexity and latency imposed on the systems. As seen in Fig. 19, the LDPC coded scheme outperforms both the ReS coded and the RSC coded arrangements. More particularly, it achieves a power gain of $3 \mathrm{~dB}$ compared to the ReS coded scheme at the CEP of $10^{-6}$. By contrast, the RSC coded scheme performs better than the ReS coded scheme only in the low $E_{b} / N_{0}$ region, namely below $7 \mathrm{~dB}$ and no 'turbo cliff' exists, even when

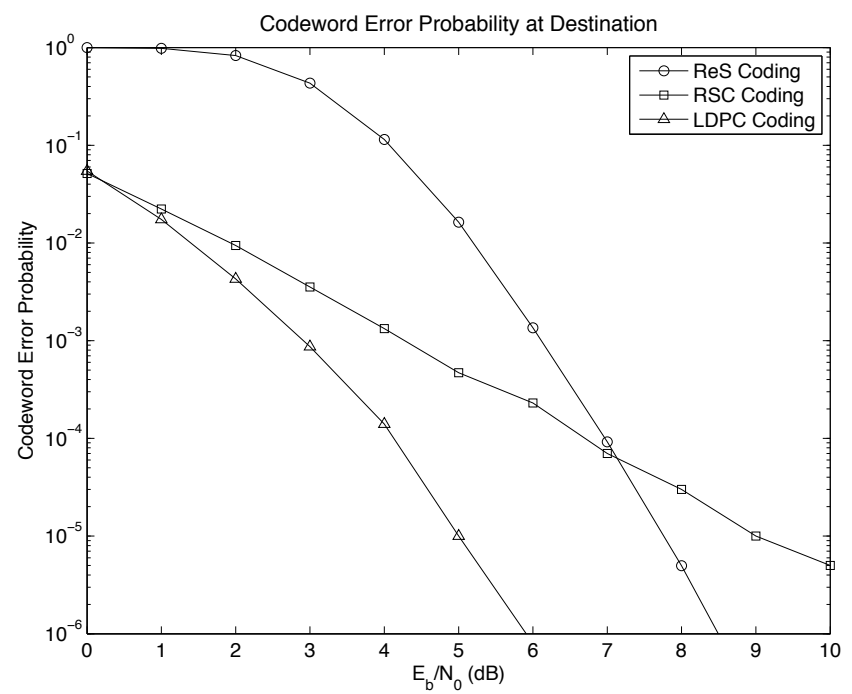

Fig. 19. Codeword decoding error probability at the destination for the RSC $(23,33)$ coded, LDPC codes and the ReS(31,20) FEC coded systems of Figs. 1 and 6 using "error-and-erasure" decoding based on the MO-RTT erasure insertion scheme for transmission over frequency-selective fading channels, when varying the interference fraction $\rho$. The results were evaluated using the parameters of $M=32, \operatorname{Re} S(31,20), L=\{1,2,3,5\}, G_{s r}=$ $G_{r d}=4 G_{s d}$, while the channels obeyed the 4-tap COST-207 model for rural area [102].

iterative detection is employed for the RSC scheme. This may be explained by the fact that the iterative detection aided RSC codes only performs well, when the packet is long, which is not the case in the cooperative scenario considered.

$b$. Iterative Decoding Aided Non-coherent Frequency Shift Keying

As a further study, the non-coherent 4-FSK is now combined with half-rate RSC convolutional coding using the octal generator polynomials $(5,7)$ in order to carry out iterative detection, where again, the other parameters of the system under consideration are provided in Table IV.

As shown in Fig. 20, the AF relaying scheme achieved a power gain of $4 \mathrm{~dB}$ in the $E_{b} / N_{0}$ region between $-4 \mathrm{~dB}$ and $10 \mathrm{~dB}$ without degrading the achievable throughput. When DF relaying is employed, a further gain of $1 \mathrm{~dB}$ may be observed in the $E_{b} / N_{0}$ region between $2 \mathrm{~dB}$ and $6 \mathrm{~dB}$ compared to the AF scheme.

\section{HARQ Design-Guidelines AND Future RESEARCH}

In this paper, we have investigated a range of factors affecting the performance of H-ARQ systems in the context of cooperative wireless communications. To conclude, our design guide-lines inferred for an H-ARQ aided cooperative system may be summarized in Fig. 21 as follows:

- Choice of channel codes: Specifically, in the system requiring a low-complexity and low latency, maximumminimum-distance block codes, such as ReS codes, may be employed. Alternatively, more sophisticated softdecision-aided convolutional codes, turbo codes [44] and LDPC codes [45], may be employed for further reducing the BER at the destination. Furthermore, the family of rateless codes [103], such as LT, SLT codes, etc., is also attractive in the context of H-ARQ systems owing to their capability of adapting to time-variant channel 


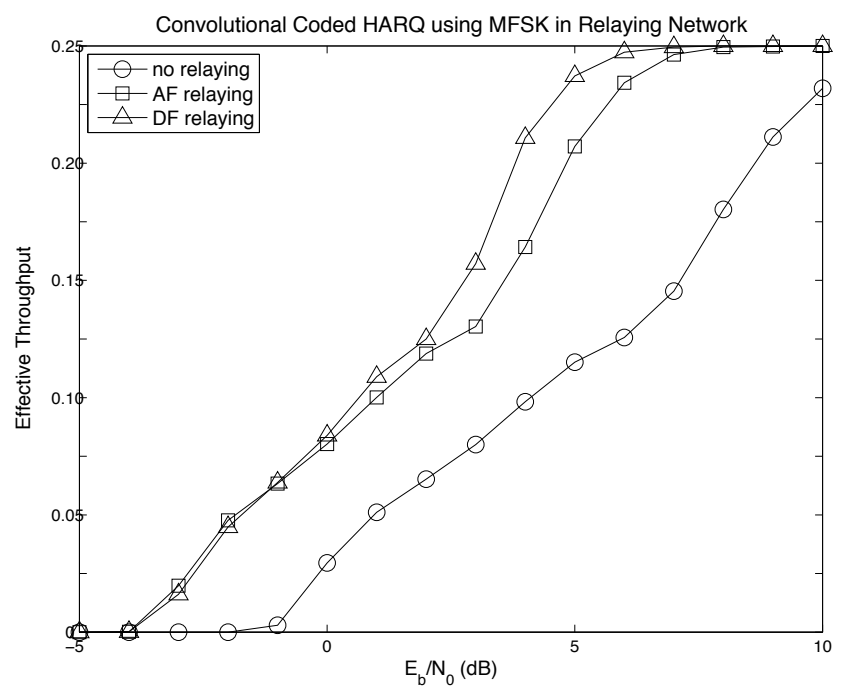

Fig. 20. The effective throughput of convolutional coded FSK aided HARQ in $\mathrm{AF}$ and DF relaying assisted networks. The receiver schematic is illustrated in Figs. 1 and 6.

conditions without requiring any channel knowledge at the transmitter.

- Detection type: In Section III, we have contrasted the family of coherent as well as non-coherent detection aided cooperative H-ARQ schemes. We demonstrated that the pilot symbol assisted coherent detection aided $\mathrm{H}$ ARQ schemes proposed in Section III.A and Section III.B reduce the effective throughput due to employing pilot symbols. By contrast, the low-complexity non-coherent schemes proposed in Section III.C avoid the pilot-related throughput loss, which partially offsets their typical $3 \mathrm{~dB}$ power loss. Their further benefit is that they dispense with channel estimation. Alternatively, the frequency bandwidth employed has to be extended in case of noncoherent FSK. Our comparisons between the perfectand imperfect-PSA coherently detected as well as noncoherent detection schemes provided in Section III.C.1.c demonstrated that the performance discrepancy between the perfect-PAS-based-coherent detection schemes and the imperfect-PSA-based-coherent schemes as well as the non-coherent arrangement increases upon increasing the Doppler frequency. In slow-fading scenarios the imperfect-PSA-based-coherent detector is preferred, while for more rapidly fading channels, differential detection relying on MSDSD might be preferred, since no throughput loss is imposed by the pilot overhead. However, differentially detected systems also exhibit a gradually eroded performance at high Doppler frequencies, which is only partially mitigated by MSDSD [57]. For moderate Doppler frequencies, the imperfect-PSA coherent and the differential non-coherent schemes perform comparably in terms of both their BER and their achievable throughput.

- ARQ-retransmission type: As mentioned in Section I, there are three main ARQ protocol types, namely Stopand-wait ARQ, Go-Back-N ARQ and Selective Repeat ARQ. Owing to their capability of improving the system throughput, Selective Repeat ARQ is preferred to its other two counterparts, which has hence been extensively employed in contemporary H-ARQ aided communication systems.

- HARQ-combining type: As detailed in Section I, the specific choice of the $\mathrm{H}-\mathrm{ARQ}$ receiver combining techniques plays a crucial role in predetermining the performance of H-ARQ schemes. By combining multiple received versions of the same transmitted packet, the CC technique [54] offers a simple power-gain based combining solution, albeit its simplicity comes at the cost having no coding gain. By contrast, the IR techniques of Section III achieve a beneficial coding gain owing to the additional parity bits gleaned from retransmissions. However, the HARQ scheme using IR has an inferior performance in comparison to that of the H-ARQ arrangement using $\mathrm{CC}$, when the systematic bits in the first transmission are corrupted by deep fades and no more systematic bits were received during the retransmission sessions [55]. For some channel codes, such as turbo codes, the residual decoding errors may occur in errorbursts, or in error-clusters within a received packet [60]. In this scenario H-ARQ schemes relying on SSR may be invoked in order to improve the attainable system performance.

- Choice of the cooperation type: The cooperation type of relay stations plays an important role in predetermining the performance of H-ARQ systems in the context of cooperative networks. As described in [58], [59], AF relaying constitutes a low-complexity cooperative scheme, where the relay simply amplifies and forwards its received signal to the destination. By contrast, DF relaying imposes a higher complexity on the relay during the decoding and re-encoding process, in the hope of improving the signal quality. Our investigations demonstrated that the relay station should invoke DF relaying, when it roams close to the source in order to avoid error propagation. By contrast, when the relay station is close to the destination, AF relaying should be activated.

- Choice of the relaying protocol: The relaying protocol, which was discussed in [38] constitutes another important aspect to be considered in the design of a cooperative system. The traditional four-phase relaying protocol attains a high diversity gain and hence an improved link quality at the cost of halving the effective throughput. The throughput loss imposed by four-phase relaying may be reduced by utilizing the successive relaying technique [104], [105]. A specific drawback of this protocol is the potential presence of interference imposed. Furthermore, network coded two-phase relaying may be employed to reduce the number of transmission phases required, albeit this is achieved at the cost of degrading the signal quality.

- Choice of the cooperative multiple access scheme: In order to mitigate the interference amongst the signals received at the destination upon increasing the number of relay nodes, the multiple-relay-assisted schemes require orthogonal subchannels for the relay node transmissions. The required orthogonality may be created in the TD, FD or SD with the aid of TDMA, FDMA, CDMA, SDMA, and so on. However, regardless of its specific 


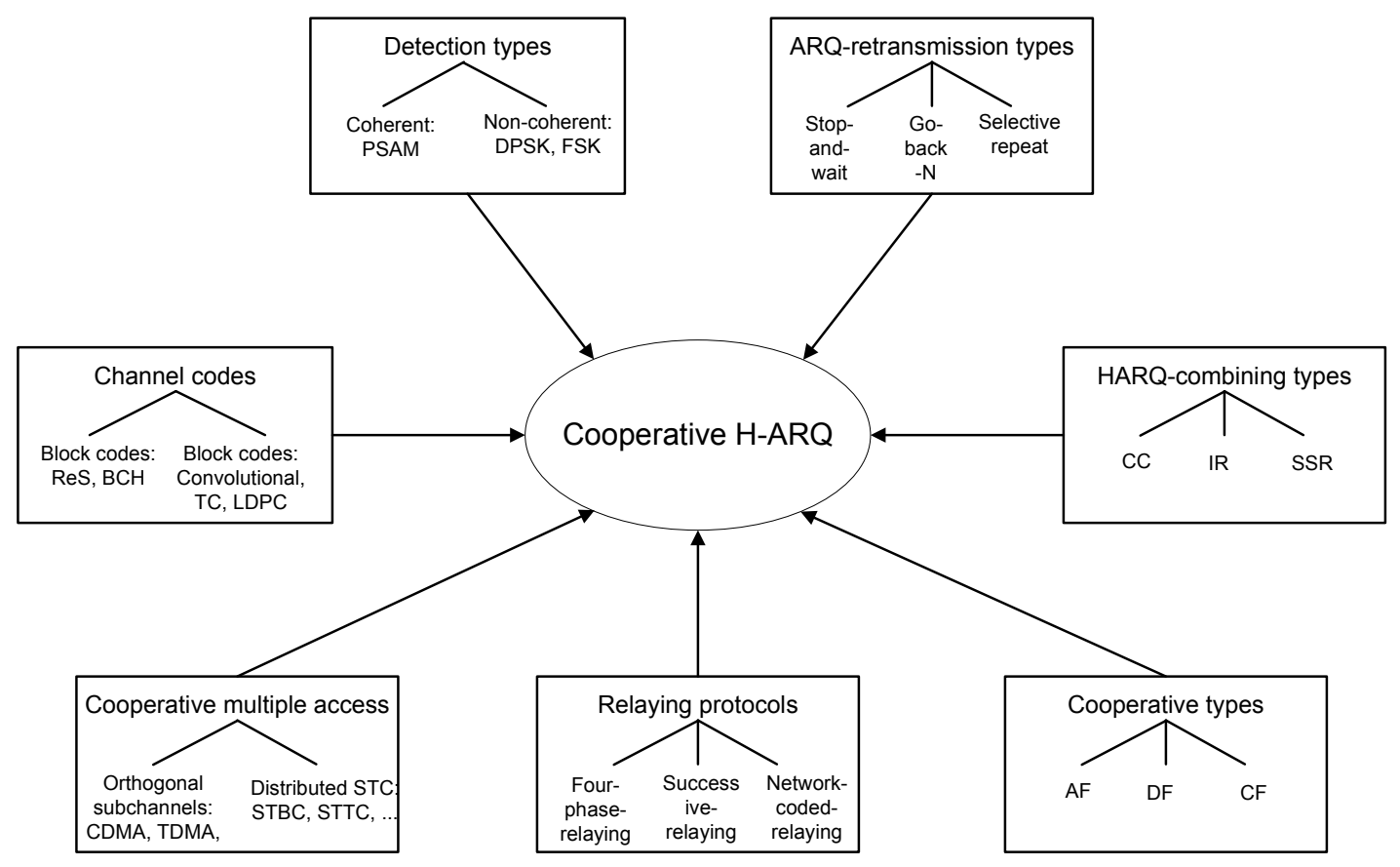

Fig. 21. Factors affecting a cooperative H-ARQ system design.

implementation, this results in a reduction of the system's spectral efficiency. A popular solution is constituted by the so-called distributed space-time coding (DSTC) philosophy [30], [106], where the relays are permitted to simultaneously transmit over the same channel by emulating a classic space-time code having co-located antenna elements. This distributed configuration is capable of retaining the DSTC system's diversity order as well as throughput [30], [106].

- Power reduction benefits: As mentioned in the introduction, it is a substantial benefit of the advances in wireless transceiver design that the achievable throughput of our transceivers has been improved in excess of three orders of magnitude in the most recent three decades. Naturally, this would imply a commensurate transmit power increase without the recent advances in signal processing, which facilitated the reduction of the per-bit energy transmitted. Quantitatively, in many cases transmit power gains in excess of $10 \mathrm{~dB}$ were achieved by our HARQ-aided transceivers, as exemplified for example in Figure 14. Furthermore, a relay station half-way between the source and destination has the potential of halving the transmit-distance, hence considerably reducing the transmit-power, depending on the pathloss exponent.

The investigations provided in this paper on the H-ARQ protocol conceived for cooperative communications may be considered a cross-layer optimization process, where the physical and MAC layers are involved. More particularly, in Section III we considered the benefits of pilot symbols as well as of channel codes on the physical layer performance of the system employing the H-ARQ protocol, which is a constituent component of the MAC layer. Then the pilot-symbol power allocation as well as channel code rate optimization was proposed in order to maximize the achievable BER and FER performance, while reducing the number of retransmission. However, in cooperative networks, the reliability of the signalling and routing messages amongst the cooperative nodes play a crucial role in the network's high-integrity operation. Therefore, in our future research we will study cross-layer operation between the physical, the MAC, the network as well as the application layers jointly for the sake of achieving further improvements.

\section{REFERENCES}

[1] L. Hanzo, H. Haas, S. Imre, D. O'Brien, M. Rupp, and L. Gyongyosi, "Wireless myths, realities, and futures: From $3 \mathrm{~g} / 4 \mathrm{~g}$ to optical and quantum wireless," Proc. IEEE, vol. 100, no. 13, pp. 1853-1888, 2012.

[2] E. C. van der Meulen, "Three-terminal communication channels," Advance Applied Probability, vol. 3, pp. 120-154, 1971.

[3] T. Cover and A. El-Gamal, "Capacity theorems for the relay channel," IEEE Trans. Inf. Theory, vol. 25, no. 5, pp. 572-584, 1979.

[4] A. Sendonaris, E. Erkip, and B. Aazhang, "Increasing uplink capacity via user cooperation diversity," in Proc. IEEE International Symposium on Information Theory, Cambridge, MA, USA, p. 156, 16-21 Aug. 1998.

[5] B. Zhao and M. Valenti, "Practical relay networks: A generalization of hybrid-ARQ," IEEE Journal on Selected Areas in Communications, vol. 23, pp. 7-18, 2005.

[6] S. Tomasin, M. Levorato, and M. Zorzi, "Analysis of outage probability for cooperative networks with HARQ," in Proc. IEEE International Symposium on Information Theory (ISIT 2007), pp. 2716-2720, 2007.

[7] C. Hasan and U. Aygolu, "An incremental relaying approach for superposition modulated cooperative transmission," in Proc. IEEE Wireless Communications and Networking Conferance (WCNC'09), Budapest, Hungary, pp. 1-6, 5-8 Apr. 2009.

[8] I. Stanojev, O. Simeone, Y. Bar-Ness, and D. H. Kim, "Energy efficiency of non-collaborative and collaborative Hybrid-ARQ protocols," IEEE Trans. Wireless Commun., vol. 8, no. 1, pp. 326-335, 2009.

[9] R. Zhang and L. Hanzo, "A unified treatment of superposition coding aided communications: Theory and practice," IEEE Commun. Surveys \& Tutorials, vol. 13, no. 3, pp. 503-520, 2010.

[10] J. S. Harsini, F. Lahouti, M. Levorato, and M. Zorzi, "Analysis of noncooperative and cooperative Type II Hybrid ARQ protocols with AMC over correlated fading channels," IEEE Trans. Wireless Commun., vol. 10 , no. 3, pp. 877-889, 2011. 
[11] Y. H. Nam, K. Azarian, H. El-Gamal, and P. Schniter, "Cooperation through ARQ," in Proc. IEEE 6th Workshop on Signal Processing Advances in Wireless Communications, pp. 1023-1027, 2005.

[12] M. Dianati, X. Ling, K. Naik, and X. Shen, "A node-cooperative ARQ scheme for wireless ad hoc networks," IEEE Trans. Veh. Technol., vol. 55, no. 3, pp. 1032-1044, 2006.

[13] I. Stanojev, O. Simeone, Y. Bar-Ness, and C. You, "Performance of multi-relay collaborative hybrid-ARQ protocols over fading channels," IEEE Commun. Letters, vol. 10, pp. 522-524, 2006.

[14] S. Savazzi and U. Spagnolini, "Design of distributed randomized orthogonal space-time coding schemes for collaborative H-ARQ," in in Proc. Conference on Signals, Systems and Computers, 2006. ACSSC '06, 2006.

[15] G. Yu, Z. Zhang, and P. Qiu, "Cooperative ARQ in wireless networks: Protocols description and performance analysis," in Proc. IEEE International Confernence on Communications (ICC '06), vol. 8, pp. 36083614, 2006.

[16] I. Krikidis, "Distributed truncated ARQ protocol for cooperative diversity networks," IET Communications, vol. 1, no. 6, pp. 1212-1217, 2007

[17] V. Mahinthan, H. Rutagemwa, J. W. Mark, and X. Shen, "Performance of adaptive relaying schemes in cooperative diversity systems with ARQ," in Proc. IEEE Global Telecommunications Conference (GLOBECOM '07), Washington DC, USA, pp. 4402-4406, 26-30 Nov. 2007

[18] K. Azarian, H. El-Gamal, and P. Schniter, "On the optimality of the ARQ-DDF protocol," IEEE Trans. Inf. Theory, vol. 54, no. 4, pp. 1718 1724, 2008.

[19] L. Le and E. Hossain, "An analytical model for arq cooperative diversity in multi-hop wireless networks," IEEE Trans. Wireless Commun., vol. 7, no. 5, pp. 1786-1791, 2008.

[20] L. Weng and R. D. Murch, "Achievable diversity-multiplexing-delay tradeoff for ARQ cooperative broadcast channels," IEEE Trans. Wireless Commun., vol. 7, no. 5, pp. 1828-1832, 2008.

[21] V. Mahinthan, h. Rutagemwa, J. W. Mark, and X. Shen, "Cross-layer performance study of cooperative diversity system with ARQ," IEEE Trans. Veh. Technol., vol. 58, no. 2, pp. 705-719, 2009.

[22] J. J. Alcaraz and J. Garcia-Haro, "Performance of single-relay cooperative ARQ retransmission strategies," IEEE Commun. Lett., vol. 13, no. 2, pp. 121-123, 2009.

[23] W. Choi, D. I. Kim, and B.-H. Kim, "Adaptive multi-node incremental relaying for hybrid-ARQ in AF relay networks," IEEE Trans. Wireless Commun., vol. 9, no. 2, pp. 505-511, 2010.

[24] W. Ni, Z. Chen, and I. B. Collings, "Hybrid ARQ based cooperative relaying in wireless dual-hop networks," in Proc. IEEE International Conference on Communications (ICC'10), pp. 1-6, 2010.

[25] R. Narasimhan, "Hybrid-ARQ interference channels with receiver cooperation," in Proc. IEEE International Conference on Communications (ICC'10), pp. 1-5, 2010.

[26] T. V. K. Chaitanya and E. G. Larsson, "Superposition Modulation Based Symmetric Relaying with Hybrid ARQ: Analysis and Optimization,” IEEE Trans. Veh. Technol., vol. 60, no. 8, pp. 3667-3683, 2011.

[27] J. N. Laneman, D. N. C. Tse, and G. W. Wornell, "An efficient protocol for realizing cooperative diversity in wireless networks," in Proc. IEEE International Symposium on Information Theory, 2001.

[28] T. E. Hunter and A. Nosratinia, "Cooperation diversity through coding," in Proc. IEEE International Symposium on Information Theory, Lausanne, Switzerland, p. 220, 30 Jun. - 5 Jul. 2002.

[29] M. Dohler, E. Lefranc, and H. Aghvami, "Virtual antenna arrays for future wireless mobile communication systems," in IEEE ICT 2002, Beijing, China, June 2002. [CD Rom].

[30] J. N. Laneman and G. W. Wornell, "Distributed space-time-coded protocols for exploiting cooperative diversity in wireless networks," IEEE Trans. Inf. Theory, vol. 49, no. 10, pp. 2415-2425, 2003.

[31] J. N. Laneman, D. N. C. Tse, and G. W. Wornell, "Cooperative diversity in wireless networks: Efficient protocols and outage behavior," IEEE Trans. Inf. Theory, vol. 50, no. 12, pp. 3062-3080, 2004.

[32] R. Nabar, H. Bolcskei, and F. Kneubuhler, "Fading relay channels: Performance limit and space-time signal design," IEEE J. Sel. Areas Commun., vol. 22, pp. 1099-1109, 2004.

[33] H. H. Sneessens and L. Vandendorpe, "Soft decode and forward improves cooperative communications," in Proc. 6th IEE International Conference on $3 G$ and Beyond, London, UK, pp. 1-4, 7-9 Nov. 2005.

[34] T. Bui and J. Yuan, "A decode and forward cooperation scheme with soft relaying in wireless communication," in Proc. IEEE 8th Workshop Signal Processing Advances in Wireless Communications (SPAWC 2007), Helsinki, Finland, pp. 1-5, 17-20 Dec. 2007.
[35] L. Xiao, T. Fuja, J. Kliewer, and D. Costello, "A network coding approach to cooperative diversity," IEEE Trans. Inf. Theory, vol. 53, no. 10, pp. 3714-3722, 2007.

[36] L. Wang, O. Alamri, and L. Hanzo, "K-best sphere detection for the sphere packing modulation aided SDMA/OFDM uplink," in Proc. IEEE Global Telecommunications Conference (GLOBECOM'08), New Orleans, LO, USA, pp. 1-5, Nov. 30 2008-Dec. 42008.

[37] H. Shan, W. Zhuang, and Z. Wang, "Distributed cooperative MAC for multihop wireless networks," IEEE Commun. Mag., vol. 47, no. 2, pp. 126-133, 2009.

[38] K. Lee and L. Hanzo, "Resource-efficient wireless relaying protocols," IEEE Wireless Commun. Mag., vol. 17, no. 2, pp. 66-72, 2010.

[39] J. M. Wozencraft and M. Horstein, "Coding for two-way channels," tech. rep., Research Laboratory of Electronics, M.I.T, 1961.

[40] J. M. Wozencraft and M. Horstein, "Digitalised communication over two-way channels," in The Fourth London Symposium of Information Theory, (London, England), Aug. 29 - Sep. 31960.

[41] R. Comroe and D. J. Costello, "ARQ schemes for data transmission in mobile radio systems," IEEE J. Sel. Areas Commun., vol. 2, pp. 472481, July 1984.

[42] S. Lin and P. Yu, "A hybrid ARQ scheme with parity retransmission for error control of satellite channels," IEEE Trans. Commun., vol. 30, pp. 1701-1719, Jul 1982.

[43] Y.-M. Wang and S. Lin, "A modified selective-repeat Type-II hybrid ARQ system and its performance analysis," IEEE Trans. Commun., vol. 31, no. 5, pp. 593-608, 1983.

[44] C. Berrou, A. Glavieux, and P. Thitimajshima, "Near shannon limit error-correcting coding and decoding: Turbo-codes.," in IEEE International Conference on Communications ICC 93. Geneva. Technical Program, Conference Record, vol. 2, pp. 1064-1070, 23-26 May 1993.

[45] R. G. Gallager, "Low-density parity-check codes," MA: MIT Press, 1963.

[46] M. Luby, "LT codes," in Proc. 43rd Annual IEEE Symposium on Foundations of Computer Science, Vancouver, BC, Canada, pp. 271280, 16-19 Nov. 2002

[47] A. Shokrollahi, "Raptor codes," in Proc. International Symposium on Information Theory (ISIT-2004), Chicago, IL, USA, p. 36, 27 June-2 July 2004

[48] L. Hanzo, J. S. Blogh, and S. Ni, 3G, HSPA and FDD versus TDD Networking: Smart Antennas and Adaptive Modulation. Wiley, 2nd ed., Feb. 2008.

[49] "IEEE standard for local and metropolitan area networks. Part 16: Air interface for fixed and mobile broadband wireless access systems," 2005.

[50] C. S. Patel and G. L. Stuber, "Channel estimation for amplify and forward relay based cooperation diversity systems," IEEE Trans. Wireless Commun., vol. 6, pp. 2348-2356, June 2007.

[51] R. Steele and L. Hanzo, Mobile Radio Communications: Second and Third-generation Cellular and WATM Systems. John Wiley - IEEE Press, 2nd ed., May 1999.

[52] C. C. Tan and N. C. Beaulieu, "Infinite series representations of the bivariate Rayleigh and Nakagami-m distributions," IEEE Trans. Commun., vol. 45, pp. 1159-1161, oct 1997.

[53] W. C. Jakes and D. C. Cox, eds., Microwave Mobile Communications. Wiley-IEEE Press, 1994.

[54] D. Chase, "Code combining: A maximum-likelihood decoding approach for combining an arbitrary number of noisy packets," IEEE Trans. Commun., vol. 33, pp. 385 - 393, may 1985.

[55] P. Frenger, S. Parkvall, and E. Dahlman, "Performance comparison of HARQ with Chase combining and incremental redundancy for HSDPA," in Proc. 54th IEEE Vehicular Technology Conference (VTC'01Fall), vol. 3, pp. $1829-1833,2001$.

[56] L. Cao and T. Shi, "Turbo codes based hybrid ARQ with segment selective repeat," Electronics Letters, vol. 40, pp. 1140 - 1141, sept. 2004.

[57] L. Hanzo, O. Alamri, M. El-Hajjar, and N. Wu, Near-Capacity MultiFunctional MIMO Systems: Sphere-Packing, Iterative Detection and Cooperation. John Wiley - IEEE Press, May 2009.

[58] A. Sendonaris, E. Erkip, and B. Aazhang, "User cooperation diversity. Part I. System description," IEEE Trans. Commun., vol. 51, pp. 1927 - 1938, November 2003.

[59] A. Sendonaris, E. Erkip, and B. Aazhang, "User cooperation diversity. Part II. Implementation aspects and performance analysis," IEEE Trans. Commun., vol. 51, pp. 1939-1948, November 2003.

[60] L. Hanzo, T. Liew, B. Yeap, R. Tee, and S. X. Ng, Turbo Coding, Turbo Equalisation and Space-Time Coding: EXIT-Chart Aided NearCapacity Designs for Wireless Channels. John Wiley \& Sons, 2nd ed., 2010. 
[61] J. G. Proakis, Digital communications. McGraw-Hill, 4th ed., 2001.

[62] B. Gedik and M. Uysal, "Impact of imperfect channel estimation on the performance of amplify-and-forward relaying," IEEE Trans. Wireless Commun., vol. 8, pp. 1468-1479, March 2009.

[63] S. Ikki, S. I. Al-Dharrab, and M. Uysal, "Exact closed-form error probability expression for cooperative diversity networks with channel estimation errors in time selective Rayleigh fading channels," in Proc. IEEE International Conference on Communications (ICC'10), Cape Town, South Africa, pp. 1-5, 2010.

[64] S. Ikki, A. Al-Dharrab, and M. Uysal, "Error probability of DF relaying with pilot-assisted channel estimation over time-varying fading channels," IEEE Trans. Veh. Technol., no. 99, 2011. Early Access.

[65] L. Hanzo, C. H. Wong, and M. S. Yee, Adaptive Wireless Transceivers: Turbo-Coded, Space-Time Coded TDMA, CDMA and OFDM Systems. Wiley-IEEE Press, 2002.

[66] Y. Wu and M. Patzold, "Performance analysis of cooperative communication systems with imperfect channel estimation," in Proc. IEEE International Conference on Communications (ICC '09), Dresden, Germany, pp. 1-6, 2009.

[67] H. Muhaidat, M. Uysal, and R. Adve, "Pilot-symbol-assisted detection scheme for distributed orthogonal space-time block coding," IEEE Trans. Wireless Commun., vol. 8, no. 3, pp. 1057-1061, 2009.

[68] S. Han, S. Ahn, E. Oh, and D. Hong, "Effect of channel-estimation error on BER performance in cooperative transmission," IEEE Trans. Veh. Technol., vol. 58, no. 4, pp. 2083-2088, 2009.

[69] X. J. Zhang and Y. Gong, "On the diversity gain in dynamic decodeand-forward channels with imperfect CSIT," IEEE Trans. Commun., vol. 59, no. 1, pp. 59-63, 2011.

[70] N. S. Ferdinand and N. Rajatheva, "Performance analysis of imperfect channel estimation in MIMO two hop fixed gain relay network with beamforming," IEEE Commun. Lett., vol. 15, no. 2, pp. 208-210, 2011.

[71] H. A. Ngo, T. D. Nguyen, and L. Hanzo, "HARQ aided systematic LT coding for amplify-forward and decode-forward cooperation," in Proc. IEEE 72nd Vehicular Technology Conference Fall (VTC 2010-Fall), pp. $1-5$, sept. 2010

[72] H. A. Ngo and L. Hanzo, "Amplify-and-forward relaying aided ReedSolomon coded Hybrid-ARQ relying on realistic channel estimation," in Proc. IEEE Vehicular Technology Conference - Spring 2010 (VTC'10-Spring), Taipei, Taiwan, 2010.

[73] L. Hanzo, Y. Akhtman, L. Wang, and M. Jiang, MIMO-OFDM for LTE, WIFI and WIMAX: Coherent versus Non-Coherent and Cooperative Turbo-Transceivers. Wiley, 2010.

[74] L. Wang and L. Hanzo, "Dispensing with channel estimation: Differentially modulated cooperative wireless communications," IEEE Commun. Surveys Tutorials, no. 99, pp. 1 -22, 2011.

[75] P. Tarasak, H. Minn, and V. K. Bhargava, "Differential modulation for two-user cooperative diversity systems," IEEE J. Sel. Areas Commun., vol. 23, no. 9, pp. 1891-1900, 2005.

[76] S. Yiu, R. Schober, and L. Lampe, "Differential distributed space-time block coding," in Proc. IEEE Pacific Rim Conference on Communications, Computers and Signal Processing (PACRIM'05), Victoria, B.C., Canada, pp. 53-56, 24-26 Aug. 2005.

[77] Y. Jing and H. Jafarkhani, "Distributed differential space-time coding for wireless relay networks," IEEE Trans. Commun., vol. 56, no. 7, pp. 1092-1100, 2008.

[78] S. M. Alamouti, "A simple transmit diversity technique for wireless communications," IEEE J. Sel. Areas Commun., vol. 16, no. 8, pp. 1451-1458, 1998.

[79] V. Tarokh, H. Jafarkhani, and A. R. Calderbank, "Space-time block codes from orthogonal designs," IEEE Trans. Inf. Theory, vol. 45, no. 5, pp. 1456-1467, 1999.

[80] Y. Jing and B. Hassibi, "Design of fully diverse multiple-antenna codes based on Sp(2)," IEEE Trans. Inf. Theory, vol. 50, no. 11, pp. 26392656, 2004.

[81] L. Wang, L. Kong, S. X. Ng, and L. Hanzo, "To cooperate or not: A capacity perspective," in Proc. IEEE 71st Vehicular Technology Conference (VTC 2010-Spring), Taipei, Taiwan, pp. 1-5, 2010.

[82] D. Divsalar and M. K. Simon, "Multiple-symbol differential detection of MPSK," IEEE Trans. Commun., vol. 38, pp. 300-308, March 1990.

[83] G. Farhadi and N. C. Beaulieu, "A low complexity receiver for noncoherent amplify-and-forward cooperative systems," IEEE Trans. Commun., vol. 58, no. 9, pp. 2499-2504, 2010.

[84] T. Himsoon, W. Su, and K. J. R. Liu, "Differential transmission for amplify-and-forward cooperative communications," IEEE Signal Process. Lett., vol. 12, no. 9, pp. 597-600, 2005.

[85] R. Annavajjala, P. C. Cosman, and L. B. Milstein, "On the performance of optimum noncoherent amplify-and-forward reception for coopera- tive diversity," in Proc. IEEE Military Communications Conference (MILCOM'05), Atlantic City, NJ, USA, pp. 3280-3288, 2005.

[86] G. Wang, Y. Zhang, and M. Amin, "Differential distributed space-time modulation for cooperative networks," IEEE Trans. Wireless Commun., vol. 5, no. 11, pp. 3097-3108, 2006.

[87] F. Oggier and E. Lequeu, "Differential distributed Cayley space-time codes," IEEE Trans. Wireless Commun., vol. 8, no. 7, pp. 3808-3814, 2009.

[88] D. G. Brennan, "Linear diversity combining techniques," Proc. IRE, vol. 47, no. 6, pp. 1075-1102, 1959.

[89] E. Agrell, T. Eriksson, A. Vardy, E. Vardy, and K. Zeger, "Closest point search in lattices," IEEE Trans. Inf. Theory, vol. 48, pp. 2201-2214, 2002.

[90] L. Hanzo, Y. Akhtman, M. Jiang, and L. Wang, MIMO-OFDM for LTE, WIFI and WIMAX: Coherent versus Non-Coherent and Cooperative Turbo-Transceivers. John Wiley \& Sons, 2010.

[91] G. Garg and F. Adachi, "Rate compatible punctured turbo-coded hybrid ARQ for OFDM in a frequency selective fading channel," in in Proc. The 57th IEEE Vehicular Technology Conference, 2003 (VTC 2003Spring), vol. 4, pp. 2725-2729 vol.4, 2003.

[92] O.-S. Shin, A. Chan, H. T. Kung, and V. Tarokh, "Design of an OFDM cooperative space-time diversity system," IEEE Trans. Veh. Technol., vol. 56, no. 4, pp. 2203-2215, 2007.

[93] J. Eberspcher, H.-J. Vgel, C. Bettstetter, and C. Hartmann, GSM Architecture, Protocols and Services. Wiley, 2009.

[94] H. A. Ngo, C. Xu, S. Sugiura, and L. Hanzo, "Space-time-frequency shift keying for dispersive channels," IEEE Signal Process. Lett., vol. 18 , no. 3, pp. 177-180, 2011

[95] W. Stark, "Coding for frequency-hopped spread-spectrum communication with partial-band interference-part i: Capacity and cutoff rate," IEEE Trans. Commun., vol. 33, no. 10, pp. 1036-1044, 1985.

[96] W. Stark, "Coding for frequency-hopped spread-spectrum communication with partial-band interference-part ii: Coded performance," IEEE Trans. Commun., vol. 33, no. 10, pp. 1045-1057, 1985.

[97] M. Pursley and W. Stark, "Performance of Reed-Solomon coded frequency-hop spread-spectrum communications in partial-band interference," IEEE Trans. Commun., vol. 33, no. 8, pp. 767-774, 1985.

[98] S. W. Kim and W. Stark, "Optimum rate Reed-Solomon codes for frequency-hopped spread-spectrum multiple-access communication systems," IEEE Trans. Commun., vol. 37, no. 2, pp. 138-144, 1989.

[99] A. J. Viterbi, "A robust ratio-threshold technique to mitigate tone and partial band jamming in coded MFSK systems," in IEEE Military Communications Conference - Progress in Spread Spectrum Communications, 1982. MILCOM 1982., vol. 1, pp. 22.4-1 -22.4-5, 17-20 1982.

[100] L.-L. Yang and L. Hanzo, "Low complexity erasure insertion in RS-coded SFH spread-spectrum communications with partial-band interference and Nakagami-m fading," IEEE Trans. Commun., vol. 50, pp. $914-925$, June 2002.

[101] L.-L. Yang and L. Hanzo, "Performance analysis of coded m-ary orthogonal signaling using errors-and-erasures decoding over frequencyselective fading channels," IEEE J. Sel. Areas Commun., vol. 19, pp. 211-221, 2001.

[102] M. Patzold, Mobile Fading Channels: Modelling, Analysis and Simulation. New York, NY, USA: John Wiley \& Sons, Inc., 2001.

[103] D. J. C. MacKay, "Fountain codes," IEE Proceedings-Communications, vol. 152, pp. 1062-1068, 9 Dec. 2005.

[104] Y. Fan, C. Wang, J. Thompson, and H. V. Poor, "Recovering multiplexing loss through successive relaying using repetition coding," IEEE Trans. Wireless Commun., vol. 6, pp. 4484-4493, Dec. 2007.

[105] B. Rankov and A. Wittneben, "Spectral efficient protocols for halfduplex fading relay channels," IEEE J. Sel. Areas Commun., vol. 25, no. 2, pp. 379-389, 2007

[106] Y. Jing and B. Hassibi, "Distributed space-time coding in wireless relay networks," IEEE Trans. Wireless Commun., vol. 5, no. 12, pp. 35243536, 2006. 


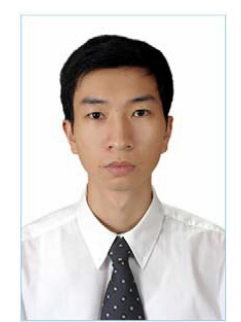

Hoang Anh Ngo received his BEng degree (with first class honour) in electronic engineering from Hanoi University of Science and Technology (HUST), Vietnam, and his MSc degree (with distinction) in wireless communications from the University of Southampton, UK. During 2008 - 2012 he worked towards his PhD degree with the Communications, Signal Processing and Control (CSPC) group, School of Electronics and Computer Science, University of Southampton, UK. He is the recipient of several academic awards from HUST, University of Southampton as well as the Engineering and Physical Sciences Research Council (EPSRC), UK. His research interests include co-located and distributed MIMO communications, space-time coding and modulation, channel modelling and cross-layer optimization.

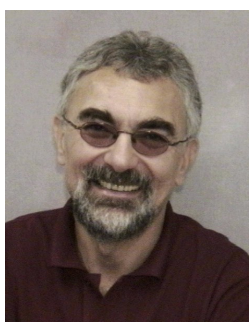

Lajos Hanzo (http://www-mobile.ecs.soton.ac.uk) FREng, FIEEE, FIET, Fellow of EURASIP, DSc received his degree in electronics in 1976 and his doctorate in 1983. In 2009 he was awarded the honorary doctorate "Doctor Honoris Causa" by the Technical University of Budapest. During his 37-year career in telecommunications he has held various research and academic posts in Hungary, Germany and the UK. Since 1986 he has been with the School of Electronics and Computer Science, University of Southampton, UK, where he holds the chair in telecommunications. He has successfully supervised $83 \mathrm{PhD}$ students, co-authored 20 John Wiley/IEEE Press books on mobile radio communications totalling in excess of 10000 pages, published 1356 research entries at IEEE Xplore, acted both as TPC and General Chair of IEEE conferences, presented keynote lectures and has been awarded a number of distinctions. Currently he is directing a 100-strong academic research team, working on a range of research projects in the field of wireless multimedia communications sponsored by industry, the Engineering and Physical Sciences Research Council (EPSRC) UK, the European IST Programme and the Mobile Virtual Centre of Excellence (VCE), UK. He is an enthusiastic supporter of industrial and academic liaison and he offers a range of industrial courses. $\mathrm{He}$ is also a Governor of the IEEE VTS. During 2008 - 2012 he was the Editor-inChief of the IEEE Press and a Chaired Professor also at Tsinghua University, Beijing. In 2012 he was awarded the European Research Council's Senior Research Fellow Grant and in 2013 the Royal Society's Wolfson Research Merit Award. For further information on research in progress and associated publications please refer to http://www-mobile.ecs.soton.ac.uk Lajos has 17 $000+$ citations. 